\title{
The quiescent core of turbulent channel flow
}

\author{
Y. S. Kwon, J. Philip, C. M. de Silva, N. Hutchins \& J. P. Monty \\ Department of Mechanical Engineering \\ The University of Melbourne, Victoria 3010, AUSTRALIA
}

(Received 22 May 2014)

The identification of uniform momentum zones in wall-turbulence, introduced by Adrian, Meinhart \& Tomkins (J. Fluid Mech., vol. 422, 2000, pp. 1-54) has been applied to turbulent channel flow, revealing a large 'core' region having high and uniform velocity magnitude. Examination of the core reveals that it is a region of relatively weak turbulence levels. For channel flow in the range $R e_{\tau}=1000-4000$, it was found that the 'core' is identifiable by regions bounded by the continuous isocontour lines of the streamwise velocity at $0.95 U_{C L}$ (95\% of the centreline velocity). A detailed investigation into the properties of the core has revealed it has a large-scale oscillation which is predominantly anti-symmetric with respect to the channel centreline as it moves through the channel and there is a distinct jump in turbulence statistics as the core boundary is crossed. It is concluded that the edge of the core demarcates a shear layer of relatively intense vorticity such that the interior of the core contains weakly varying, very low-level turbulence (relative to the flow closer to the wall). Although channel flows are generally referred to as 'fully turbulent', these findings suggest there exists a relatively large and 'quiescent' core region with a boundary qualitatively similar to the turbulent/non-turbulent interface of boundary layers, jets and wakes.

\section{Introduction}

The pursuit of an improved understanding of wall-turbulence has seen comparisons and exchange of results between experiments in enclosed geometries (e.g. pipes and rectangular ducts) and 'external' geometries, i.e. boundary layers. The interchangeability of these different flows is limited by differences in the outer flow. Balakumar \& Adrian (2007), Monty et al. (2009) and Jiménez et al. (2010) are some of the recent articles comparing and citing differences between pipes, channels and boundary layers. A seemingly universal view and fundamental difference is that pipes and channels are 'fully turbulent', while boundary layers entrain non-turbulent, potential flow from the free-stream (Davidson 2004). This paper presents a slightly alternative view.

In free-shear and boundary layer flows, the existence of an interface between turbulent flow and the non-turbulent free-stream is clear and can be rather easily identified. The foundations for interface studies are presented in the seminal works of Townsend (1948, 1956) and Corrsin \& Kistler (1955), and more recent progress is presented in Hunt et al. (2011) and da Silva et al. (2014). In turbulent boundary layers, Corrsin \& Kistler (1955) and Kovasznay et al. (1970) are two of the earlier studies wherein they have defined the turbulent/non-turbulent (TNT) interface by vorticity changes in the outer region. This method of defining the interface is common for boundary layers as well as studies in jets and wakes (e.g. Bisset et al. 2002; da Silva \& Pereira 2008). At the TNT interface, there is a layer termed the 'viscous superlayer', first proposed by Corrsin \& Kistler (1955). In the superlayer, vorticity is transmitted from the turbulent into the non-turbulent region by viscous diffusion. Recent studies also suggest the existence of the 'turbulent sublayer', 
flanked by the viscous superlayer and the turbulent region, across which there is a sharp change in vorticity (e.g. da Silva et al. 2014). Across TNT interfaces, a peak in vorticity corresponding to a jump in streamwise velocity can be observed as shown in recent experimental studies of turbulent boundary layers using particle image velocimetry (e.g. Semin et al. 2011; Chauhan et al. 2014). However, it should be noted that there are some cases where no vorticity peaks are observed (da Silva et al. 2014). Hence, a vorticity peak is just an indication of a vorticity layer and is not a necessary condition for the presence of a TNT interface.

Fully developed turbulent channel flows are generally considered 'fully turbulent' due to the constriction of the opposing walls. There are relatively few studies focussing on the far outer region of channel flows and the authors could not find any previous work on internal interfaces in turbulent channel flows, at least not in homogeneous media (Lofquist (1960) and Silva \& de Lemos (2003) document internal interfaces in channels with inhomogeneous media). However, Dean \& Bradshaw (1976) present a different conceptual view where channel flows consist of interacting boundary layers originating from either wall of the channel. That investigation involved experiments where the boundary layer originating from one wall was 'tagged' with a series of heated wires such that temperature could be used to detect the shear layer as it evolved. Conditional statistics of the identified internal shear flows originating from either wall were acquired and compared with boundary layer statistics at comparable Reynolds number. They concluded that the channel flow is well approximated by the superposition of two internal boundary layers growing from each wall. Any interaction between the two internal boundary layers was found to be confined within $\pm 0.2 H$ of the centreline, where $H$ is the full channel height. Antonia et al. (1992) employed a similar strategy of 'tagging' the fluid elements near one wall by heating a section of the wall both experimentally and using direct numerical simulation (DNS) at low Reynolds numbers. By detecting the trace of heated fluid elements, they found that the 'tagged' elements can penetrate into the near-wall region of the opposite wall. However, the overall contribution from these thermal excursions to the Reynolds shear stress and the spanwise vorticity was found to be negligible due to the low frequency of such excursions.

Regarding interaction across the centreline, Sabot \& Comte-Bellot (1976) also found that bursts associated with both walls co-exist and a burst from one wall can extend into the opposing half of the pipe up to a distance of $1.3 R$ from the wall (where $R$ is the pipe radius). Teitel \& Antonia (1990) present similar findings for channel flows, where an analysis of Reynolds stress showed that Q2 events originating from a given half of the channel can extend into the opposite half. Interestingly, Teitel \& Antonia (1990) also find that during such events there is an anti-symmetry in the flow by examining the phase difference between the Fourier components of the wall-normal velocity signals on either sides of the channel centreline. Similarly, Jiménez et al. (2010) found that the large-scale ejections from a given half of the channel can extend deep into the opposite half by examining the fractional quadrant contributions to the Reynolds shear stress. In addition, they computed the correlations of the large-scale streamwise and wall-normal velocity fluctuations to show that the coherence of the large-scale structure of wall-normal velocity persists across the central $50 \%$ of the channel and the large-scale streamwise velocity fluctuations are anti-symmetric in turbulent channel.

If internal flows can be approximated by the interaction of boundary layers growing from opposing walls, it is possible that there are internal layers in channel flows similar to boundary layers, although these are not a necessary condition for the validity of such an approximation. As such, this paper seeks to separate a channel flow into two regions: (i) a so-called 'quiescent core' in the outer region having approximately uniform momentum; 
(ii) a turbulent shear flow closer to the wall, presumably originating from and attached to the wall. Throughout this paper, the term 'quiescent' is not used as a synonym for the term 'non-turbulent'. It is used to describe the state of relatively low turbulence levels as compared to the rest of channel flow. In this regard, the 'quiescent core' is fundamentally different to the potential flow.

Beginning from the work of Meinhart \& Adrian (1995), it is known that the instantaneous streamwise velocity profile in a wall-bounded flow has steps of uniform velocity, or has uniform momentum zones. The wall-normal extent of these zones become smaller as we approach the wall. The corresponding vorticity field shows that instantaneously vorticity is mostly lumped into compact regions which are predominantly located along the edges of these uniform momentum zones (even though one can also observe them more dispersed towards the wall). Our approach here is to isolate the largest uniform momentum zone, using a method which is an improvisation of that originally introduced by Adrian et al. (2000) (as outlined in section 3). Since this uniform momentum zone covers the bulk of the central region of the channel, it is termed as the 'core' region. Furthermore, we analyse the properties of the core in section 4 and the turbulent levels across the boundary of the core in section 5, which then suggest that the core is quiescent. This leads us to draw similarities between the non-turbulent and turbulent regions in free-shear/boundary layer flow, and the quiescent core and the region closer to the wall in channel flow. We also show that due to conflicting influences from the top and the bottom walls, the core 'flops' inside the channel with a predominantly anti-symmetric mode (similar to the flopping of a non-turbulent jet with its symmetric/anti-symmetic modes; e.g. Drazin \& Reid 1981). Finally, in section 6 we comment on the general uniform momentum zones and finish with conclusions in section 7 .

Throughout this paper, $x, y$ and $z$ denote the streamwise, spanwise and wall-normal coordinates respectively. $U, V$ and $W$ denote the corresponding components of total instantaneous velocity. Velocity quantities with a lower case letter represent the fluctuations about time-averaged velocity (e.g. $u=U-\bar{U}$ ).

\section{Experimental set-up}

The experiments are conducted in the channel flow facility at the Walter Bassett Aerodynamics Laboratory located at the University of Melbourne. Full details are provided in Monty (2005). The working section measures $22 \mathrm{~m}$ in length with a cross section of $1170 \times 100 \mathrm{~mm}^{2}$. The facility is able to generate a maximum flow rate of approximately $3.6 \mathrm{~m}^{3} / \mathrm{s}$, which equates to a bulk velocity of approximately $30 \mathrm{~m} / \mathrm{s}$ in the working section. A glass section is built into the existing facility, as shown in figure 1, to enable optical access, which is necessary for conducting the particle image velocimetry (PIV). The modifications consisted of a glass floor and glass side walls with a length of $1400 \mathrm{~mm}$ in the streamwise direction. The roof consisted of a removable glass section with a length of $500 \mathrm{~mm}$ in the streamwise direction. The PIV measurements are conducted approximately $18 \mathrm{~m}$, or $360 h$, where $h$ is the channel half-height, downstream of the inlet to the working section. This ensures that the flow is fully developed for the first and the second order statistics (Monty 2005).

A two-dimensional planar PIV experiment is set up, as shown in figure 1. The planar PIV imaging system consists of four PCO 4000 cameras $(4008 \times 2672$ pixels, $2 \mathrm{~Hz})$ equipped with $105 \mathrm{~mm}$ Sigma lenses. This setup enables us to obtain a field of view of $0.6 \mathrm{~m}(12 h)$ in the streamwise direction and $0.1 \mathrm{~m}(2 h)$ in the wall-normal direction, with a magnification such that one pixel size corresponds to approximately $45 \mu \mathrm{m}$. The flow is seeded with polyamide particles with a mean particle diameter of $1 \mu \mathrm{m}$ and illuminated using a dual 


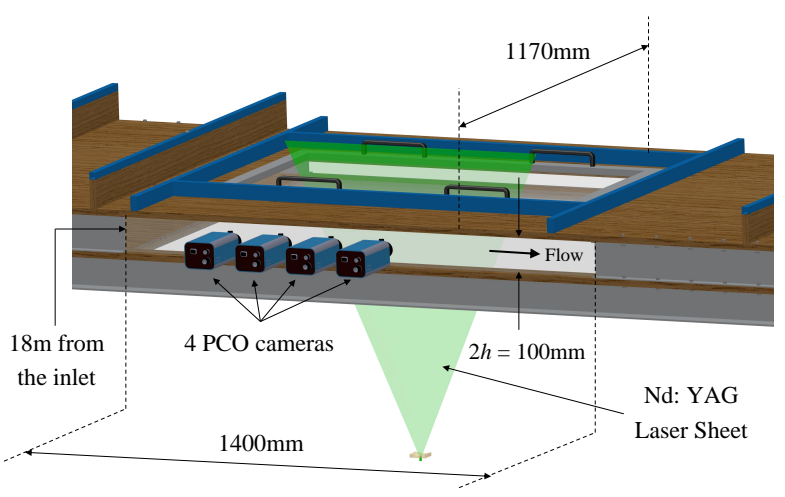

FIGURE 1. Experimental setup for 2D planar PIV.

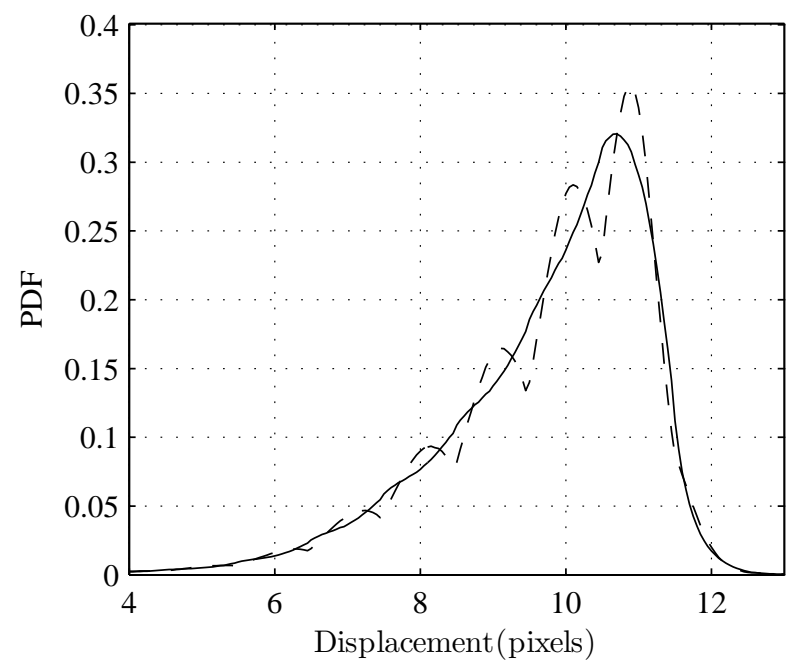

FiguRE 2. PDF of pixel displacement for $U$. Dashed and solid lines indicate the PDF of pixel displacement before and after applying the histogram equalisation technique, respectively.

cavity Big Sky Nd:YAG laser which delivers 180mJ/pulse. In order to capture a large field of view, magnification of the seeding particles has to be compromised, which results in a peak-locked dataset. To overcome this, a histogram equalisation technique introduced in Roth \& Katz (2001) is used to reduce the peak-locking effect. Figure 2 shows probability density functions (PDF) of pixel displacement for $U$ before and after implementing the histogram equalisation technique. Before the correction, the pixel displacements for $U$ are biased towards the integer values but the peak-locking effect is minimised after applying the histogram equalisation technique.

Four data sets are obtained at the nominal friction Reynolds numbers of $R e_{\tau}=1000$, 2000, 3000 and 4000, by adjusting the bulk velocity, $U_{b}$. $\left(R e_{\tau}=h U_{\tau} / \nu\right.$ where $U_{\tau}=\sqrt{\tau_{w}} / \rho$ is the friction velocity, $\tau_{w}$ is the wall shear-stress, $\rho$ is the fluid density and $\nu$ is the kinematic viscosity). Parameters of the experiment are summarised in table 1. Prior to each measurement six pressure tappings located approximately $2 \mathrm{~m}$ apart are used 


\begin{tabular}{cccccc}
\hline \multicolumn{2}{c}{$e_{\tau}$} & $\begin{array}{c}\nu / U_{\tau} \\
(\mathrm{m})\end{array}$ & $\begin{array}{c}\text { Interrogation size } \\
\text { (Pixels) }\end{array}$ & $\begin{array}{c}\text { Interrogation size } \\
\text { (Wall units) }\end{array}$ & Number of images Symbol \\
\hline $1030(\approx 1000)$ & $4.886 \times 10^{-5}$ & $16 \times 16$ & $15 \times 15$ & 1000 & \\
$2100(\approx 2000)$ & $2.381 \times 10^{-5}$ & $16 \times 16$ & $30 \times 30$ & 1210 & $\square$ \\
$3090(\approx 3000)$ & $1.635 \times 10^{-5}$ & $16 \times 16$ & $44 \times 44$ & 735 & $\triangle$ \\
$3965(\approx 4000)$ & $1.274 \times 10^{-5}$ & $16 \times 16$ & $56 \times 56$ & 588 & $\nabla$ \\
\hline
\end{tabular}

TABLE 1. Summary of measurement details.

to obtain the streamwise pressure gradient, which enables determination of the friction velocity, $U_{\tau}$.

The experimental data are processed using an in-house PIV software, details of the algorithms are given in de Silva et al. (2012). The cross-correlation is performed with an interrogation window size of $16 \times 16$ pixels (about $0.72 \times 0.72 \mathrm{~mm}$ ). Further details on the PIV experiment are given in table 1 . It should be noted that since the same optical system is used for the four Reynolds numbers considered, a drop in spatial resolution occurs as the Reynolds number is increased. However, all analyses focus on the outer region of the flow where spatial resolution effects will not change the conclusions of this study. Fewer images were obtained at $R e_{\tau}=3000$ and 4000 due to time constraints during the experiment. However, these numbers are sufficient to obtain converged first and second order statistics at each Reynolds number and these statistics were validated against prior measurements and DNS databases (see Appendix A).

\section{Identification of the channel core}

Employing the concept of uniform momentum zones introduced by Meinhart \& Adrian (1995), we shall define the core of a channel flow to be the zone of highest uniform momentum; that is, the region where the streamwise velocity varies only weakly about a value close to the mean centreline velocity, $U_{C L}$. To identify the velocity threshold that defines the boundary of this approximately uniform momentum zone, a PDF of instantaneous velocity is used, similar to that employed in Adrian et al. (2000). The method is best explained with reference to figure 3. Firstly, a subregion of the channel of the streamwise length $1.2 \mathrm{~h}$ is taken as illustrated in figure 3(a). Figure 3(b) shows histograms of $U / U_{C L}$ constructed from the velocity field shown in figure 3(a) using the upper and lower halves of the channel. The PDF of $U$ is, of course, sensitive to the streamwise extent of the flow considered. Considering an infinite length domain would result in a PDF with a single peak corresponding to the centreline velocity (figure 2). The PDF constructed from any finite domain of length $\leqslant O(h)$ may contain local maxima, which Adrian et al. (2000) interpret as 'local' uniform momentum zones. Therefore, the peaks in the PDF of $U$ in figure 3(b) are the consequence of local uniform momentum zones in figure 3(a). To illustrate this, each local uniform momentum zone and the corresponding peak in the PDF are labelled (T1, T2 and T3 for the upper half and B1 and B2 for the lower half). The black contour lines in figure 3(a) correspond to the velocity values at the valleys between the peaks in figure 3(b). The PDFs for the upper and lower halves are shown separately to enable the direct comparison to Adrian et al. (2000) where they use the PDF of $U$ to identify uniform momentum zones in turbulent boundary layers. It should 
(a)
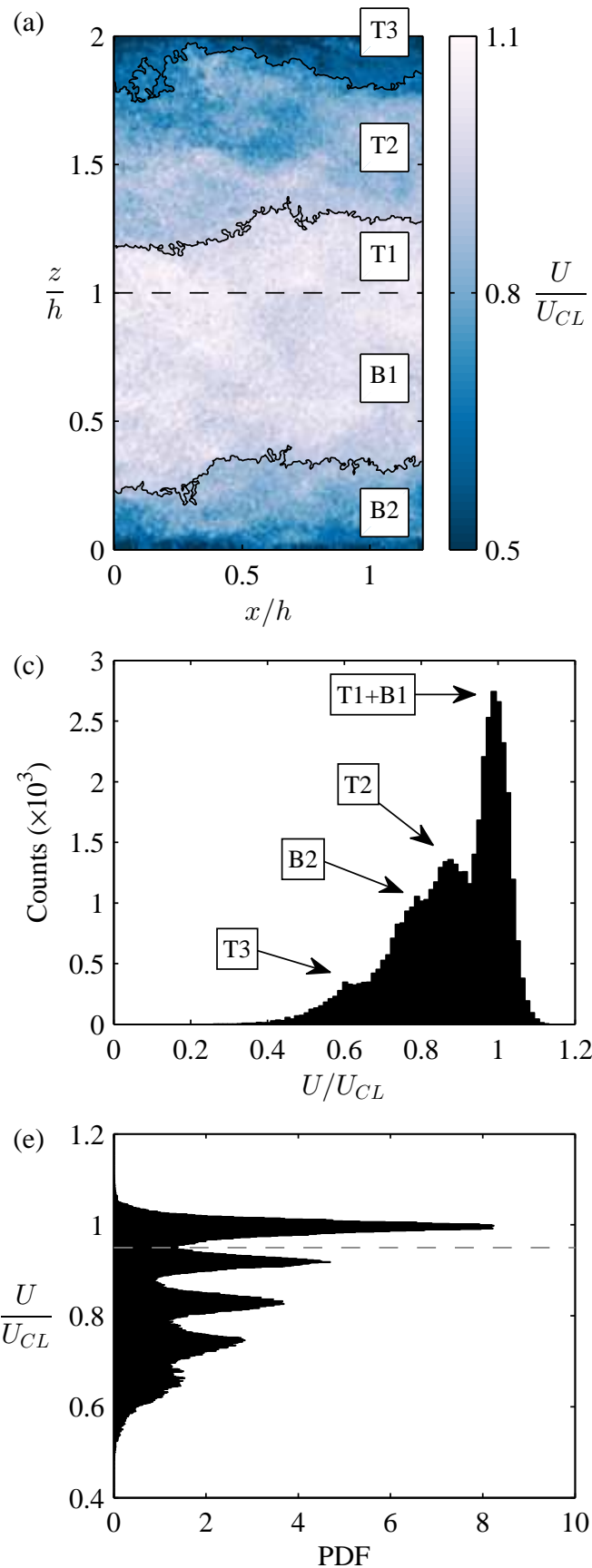

(b)
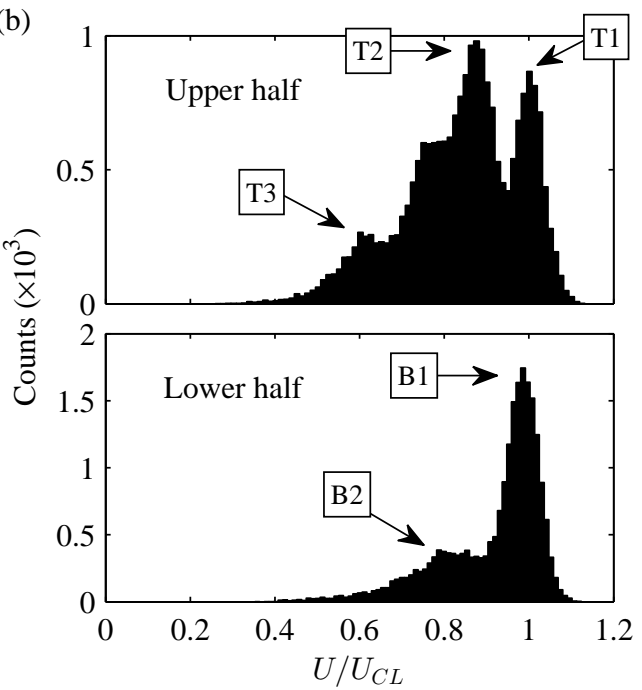

(d)

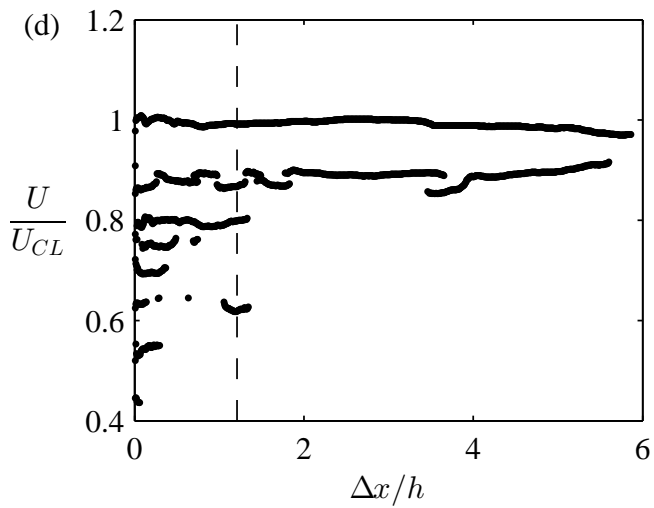

(f)

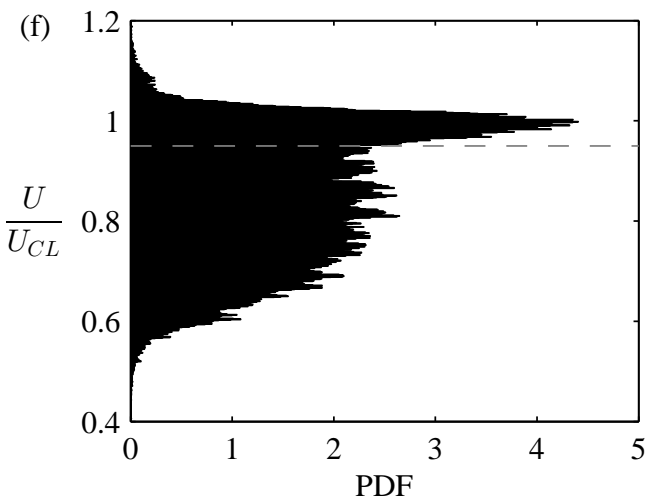

Figure 3. (a) Contour plot of $U / U_{C L}$ of an example vector field subregion $(\Delta x=1.2 h)$ from a PIV frame at $R e_{\tau}=1000$. The dashed line indicates the channel centreline. (b) Histograms of $U / U_{C L}$ constructed using the upper and lower halves of the example field shown in (a). (c) Histogram of $U / U_{C L}$ constructed using the entire example field shown in (a). (d) Plot of the modal velocities (peaks) in histograms of $U / U_{C L}$ in the same PIV frame as a function of the considered streamwise domain. The black dashed line indicates the domain of $\Delta x=1.2 h$ in the streamwise direction employed to find the peaks, so the modal velocities on this line correspond to peaks in the histogram shown in (b). (e) PDF of the modal velocities found in all PIV fields at $R e_{\tau}=1000$. The gray dashed line indicates $U=0.95 U_{C L}$. (f) $\mathrm{PDF}$ of the modal velocities found using DNS at $R e_{\tau}=1000$. 
be noted that these distributions are similar to those documented by Adrian et al. (2000) in a $1.2 \delta$ long subregion of a boundary layer, which they used for the identification of 'modal velocities'. A 'modal velocity' is identified by a local peak in the PDF of $U$. For example, in figure 3(b), there are a number of peaks at various velocities (e.g. in the upper half of the channel there are peaks at $U / U_{C L} \approx 1.0,0.9$ and 0.6 , labelled $\mathrm{T} 1, \mathrm{~T} 2$ and T3 respectively), but the peak common to all is at $U / U_{C L} \approx 1$. This peak represents the channel core. Figure 3(c) displays the histogram over the full height of the channel (i.e. the superposition of the two PDFs shown in figure $3 \mathrm{~b}$ ). Notice that its peaks give all uniform momentum zones over the full channel height. While it is possible to identify the core through analysis of the PDF of $U$ only, such an identification is sensitive to the length of domain from which the PDF is calculated. Therefore, we seek a more robust approach through the analysis of the modal velocities where the length of the channel, or the streamwise domain used for constructing the PDF is varied.

Figure 3(d) shows how the modal velocities (peaks in the PDF) vary as a function of the streamwise domain considered up to the maximum domain of $6 h$. To arrive at figure 3(d), we use the same PIV frame as that from which figure 3(a) was extracted and PDFs of $U / U_{C L}$ were calculated over the full channel height (as exemplified in figure 3c). For reference, the vertical dashed line (in figure $3 \mathrm{~d}$ ) indicates the $1.2 \mathrm{~h}$ domain, at which the PDF of $U / U_{C L}$ exhibits four peaks. These correspond to the peaks in the PDF that can be observed in figure 3(c) (shown by the four arrows corresponding to four modal velocities). In this particular example subregion, for streamwise domains greater than approximately $2 h$, the number and $U / U_{C L}$ values of the peaks remain relatively constant over an extended streamwise domain, indicating that they are robust uniform momentum zones over this streamwise domain. For the example above, we have considered only one subregion of the velocity field. If the procedure above is repeated for all fields, one obtains a set of modal velocities (PDF peaks) as the streamwise domain is varied, for each field. A PDF of the modal velocities so obtained is shown in figure 3(e). It shows the likely streamwise velocity values of local momentum zones in turbulent channel flow at $R e_{\tau}=1000$. The dominant peak at $U=U_{C L}$ in figure $3(\mathrm{e})$, indicates the core of the channel flow. Again, the local minima in modal velocity distribution below this peak occurs at $0.95 \pm 0.01$. There are a series of secondary peaks at lower velocities that should be considered spurious. They are due to the residual effects of the 'peak-locking' problem discussed earlier, despite attempts to mitigate it, rather than a consequence of statistically preferred modal velocities. Although spurious peaks appear in the PDF of modal velocities, the actual degree of peak-locking is not severe enough to create spurious uniform momentum zones as demonstrated by the corrected PDF of $U$ in figure 2. Instead, the peak-locking effect is only able to cause streamwise velocity values of the real uniform momentum zones (modal velocities) to be biased towards velocities corresponding to integer pixel displacements, creating the spurious peaks in the modal velocity distribution. To illustrate that the primary peak and the minima at $U / U_{C L}=$ 0.95 are robust features of the channel flow, the same procedure was applied to DNS data at $R e_{\tau} \approx 1000$ and the result plotted in figure $3(\mathrm{f})$. The DNS data are from the channel flow simulation by del Alamo et al. (2004). The secondary peaks do not exist in the simulation, as expected, yet the primary maxima and its nearest minima remain at the same $U / U_{C L}$ values. The core identification threshold of $U / U_{C L}=0.95$ was found to be applicable for all Reynolds numbers considered within the error of the identification scheme and the experimental data.

In figure 4 , the channel cores identified using the boundary value of $U / U_{C L}=0.95$ are highlighted on top of contours of instantaneous streamwise velocity fields (from PIV). From here on, the superscript ' + ' indicates the normalization by the viscous scales, $U_{\tau}$ 

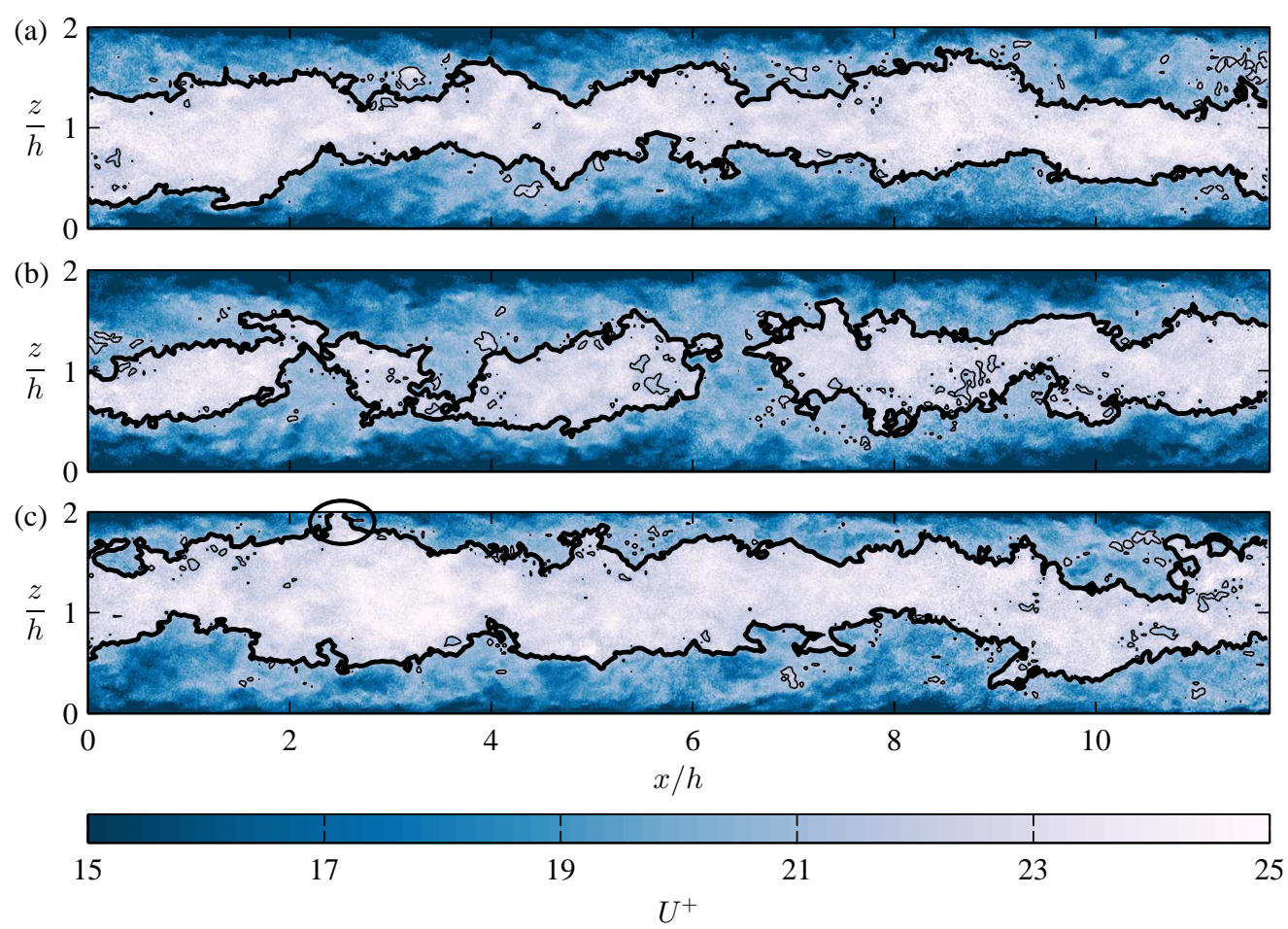

FiguRE 4. Examples of instantaneous $U$ fields from PIV at $R e_{\tau}=1000$. Black lines indicate the contour lines of $U=0.95 U_{C L}$. Thicker lines indicate the core boundaries. (a) The core extends throughout the whole field of view. (b) The core is sometimes discontinuous. (c) The core boundary can reach very close to the wall (highlighted by the ellipse).

(velocity) and $\nu / U_{\tau}$ (length). The core region is defined as the region bounded by the continuous boundaries of the uniform momentum zone. The holes where $U<0.95 U_{C L}$ that exist in the uniform momentum zone (the core) are ignored and still treated as the part of the core region, and the islands of fluid with $U>0.95 U_{C L}$ outside of these boundaries are not treated as the core region. It is observed that the regions enclosed by the boundaries defined by contour lines of $U / U_{C L}=0.95$ have roughly uniform streamwise velocity that is distinct from the rest of the channel flow. Figure 4(a) shows that the channel core shifts up and down about the channel centreline and has varying thickness. The core can sometimes be discontinuous as shown in figure 4(b), which is to be expected in a confined flow. Surprisingly, the channel core boundary can even reach very close to the wall in extreme instances, an example of which is shown circled in figure 4(c). The DNS data gives access to the wider velocity field, permitting observation of the core in both the streamwise- and spanwise-wall-normal planes as shown in figure 5 . It is interesting to note the relatively smaller wavelength of the core boundary in the spanwise-wall-normal plane (figure $5 \mathrm{~b}$ ).

Figure 5(c) presents a three-dimensional view of the core boundary, obtained by plotting the isosurface of $U=0.95 U_{C L}$ from a subregion of the DNS field (approximately $1 / 6$ of the full simulation box). With the benefit of information in the third-dimension, the core has the form of a corrugated sheet, which spans the entire channel. The core contains large-scale streamwise streaky features that appear to extend about $4 h$ in the streamwise direction and have a spanwise spacing of about $h$. These streaky features can come close to the wall as indicated by the red coloured regions in figure 5(c) (the bright 

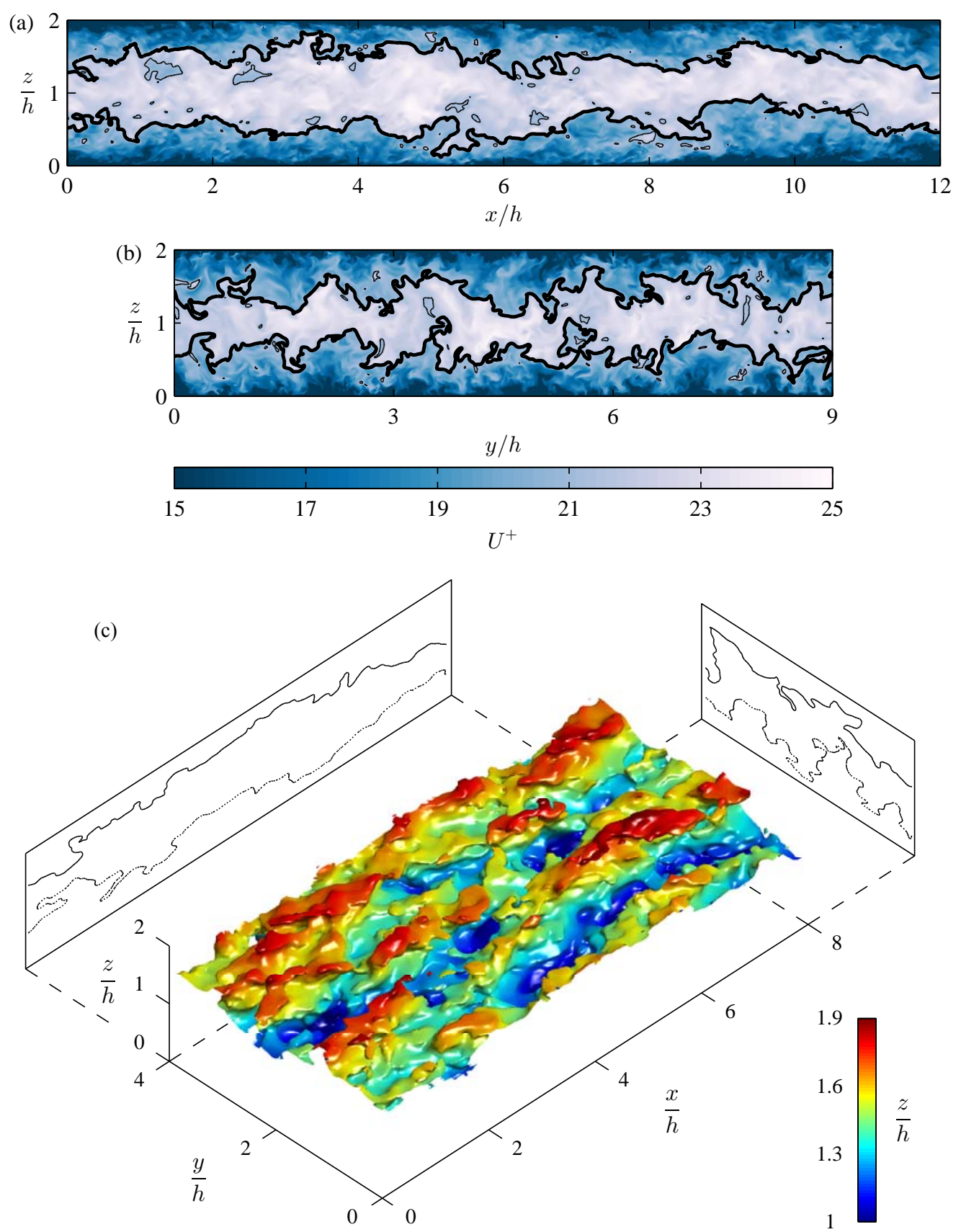

Figure 5. Examples of instantaneous $U$ fields from DNS data of del Alamo et al. (2004) at $R e_{\tau}=1000$ (a) in the streamwise-wall-normal plane (b) in the spanwise-wall-normal plane. Black lines indicate the contour lines of $U=0.95 U_{C L}$. Thicker lines indicate the core boundaries. (c) Isosurface of $U=0.95 U_{C L}$ obtained from DNS data of del Alamo et al. (2004). Only the upper surface of the core is shown for clarity. Colour indicates the wall-normal coordinate of the isosurface. The $2 \mathrm{D}$ projected views show both the upper and lower core boundaries at the end of the domain (at $y=4 h$ for the streamwise-wall-normal view and at $x=8 h$ for the spanwise-wall-normal). 

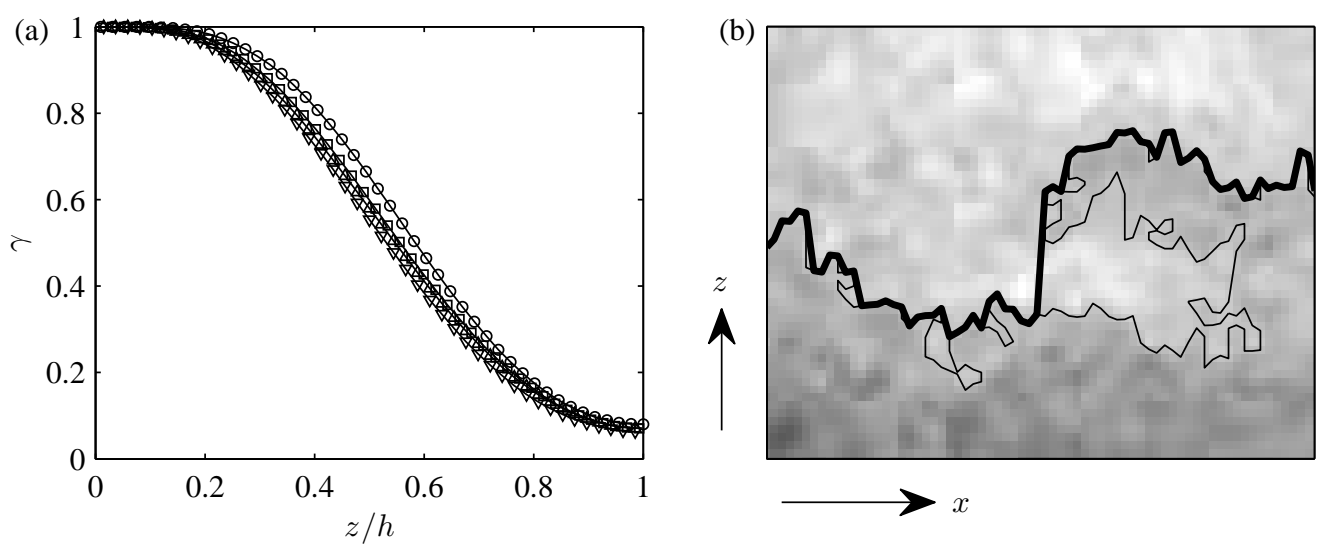

FiguRE 6. (a) Intermittency, $\gamma$, at various wall-normal positions. Symbols given in table 1 . (b) Example of 'inner' enveloped upper core boundary (thick line). The thin line indicates the un-enveloped core boundary $\left(0.95 U_{C L}\right.$ contour).

red colour indicating a proximity of $\sim 0.1 h$ to the wall). Very close to the upper wall $(z=2 h)$, the high speed core spreads in the spanwise direction due to the presence of the wall which leads to the flat tops (red coloured regions) of the streaky core boundary.

\section{Characterisation of the core}

With a defined core, an 'intermittency' of channel flow can now be defined in the same manner as has been done in boundary layers and free shear flows (e.g. Townsend 1948, 1956; Corrsin \& Kistler 1955; Kovasznay et al. 1970). The intermittency, $\gamma$, is determined by the proportion of time that the channel flow spends outside of the core at various wallnormal positions. Figure 6(a) shows the intermittency profile across the channel. Up to $z / h \approx 0.1$, the flow spends almost no time in the core, while at the centreline, the flow is outside of the core for roughly $7 \%$ of the time. The latter indicates times when the core is discontinuous or the core completely crosses the channel centreline. A Reynolds number trend is also observed, where the flow spends more time in the core at higher $R e_{\tau}$ for any wall-distance (alternatively the horizontal shift of the intermittency profiles in figure 6(a) with increasing Reynolds number). This is likely due to increased core thickness at higher $R e_{\tau}$, which is to be expected because $z /\left.h\right|_{U=0.95 U_{C L}}$ decreases with Reynolds number (albeit weakly). However, it is difficult to predict the asymptotic behaviour of the core (without data at higher Reynolds numbers) because the velocity threshold of the highest uniform momentum zone may be a weak function of Reynolds number also.

Although this figure is sensitive to the velocity threshold, it provides an illustration of the time spent inside and outside the core (as defined by the velocity contour $0.95 U_{C L}$ ), which is helpful to keep in mind as we examine zonal statistics below.

In order to understand how the turbulent channel flow differs in the core compared with outside of the core, zonal mean statistics are computed (Kovasznay et al. 1970, documented similar statistics for boundary layers). Figures 7(a) and (b) show the zonal mean profiles of the streamwise velocity. For a given $z / h, \tilde{U}$ and $\hat{U}$ are the mean streamwise velocities conditioned on whether that wall-normal location is inside or outside of the core, respectively. Throughout the paper, a tilde over a variable indicates the zonal mean inside the core and a hat indicates the zonal mean outside the core. The global time-averaged streamwise velocity $\bar{U}$ is also plotted as a reference. The weighted average 

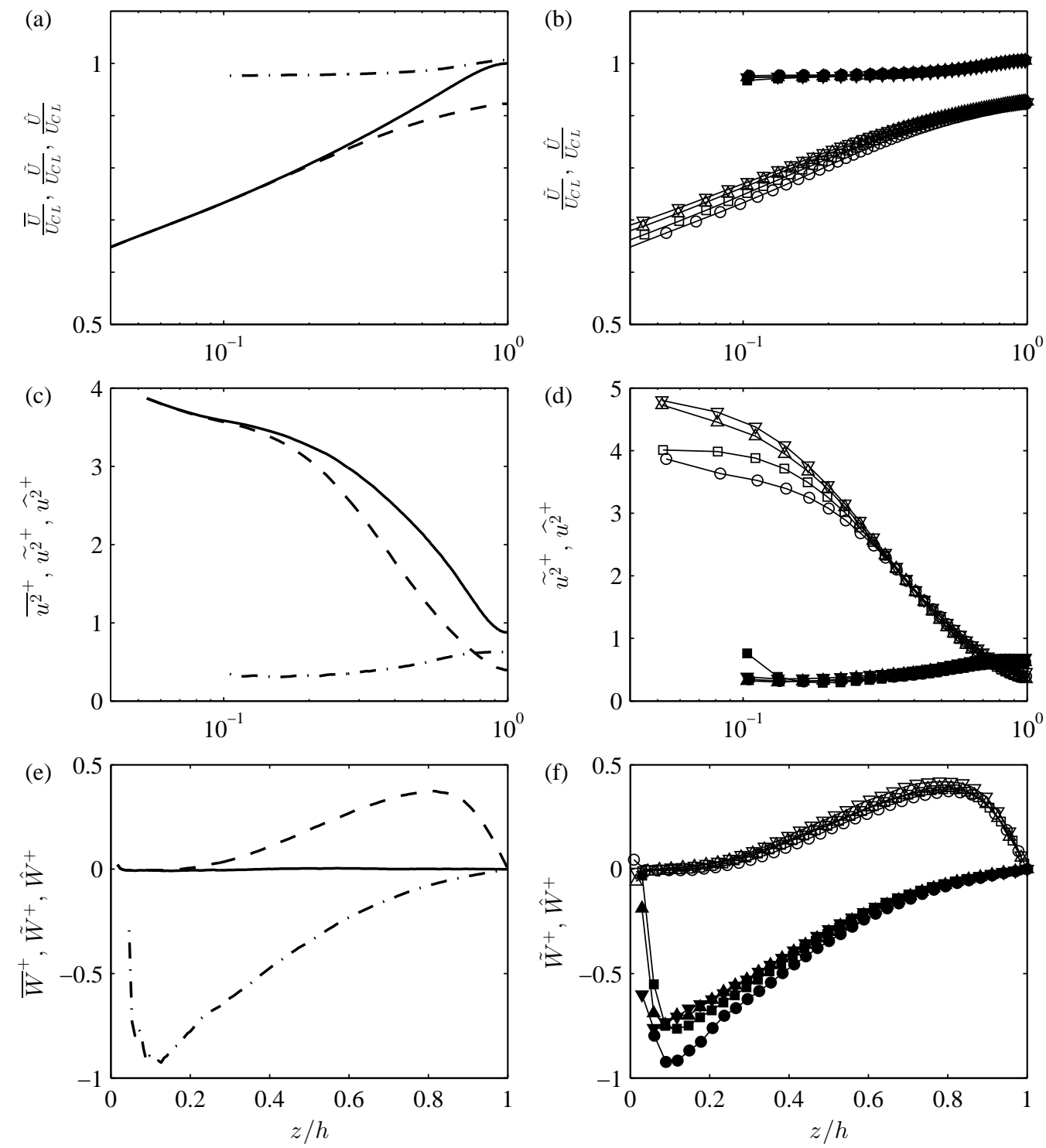

FiguRE 7 . Zonal mean streamwise velocity profiles at (a) $R e_{\tau}=1000$, and (b) all $R e_{\tau}$. zonal mean streamwise turbulent intensity profiles at (c) $R e_{\tau}=1000$, and (d) all $R e_{\tau}$. zonal mean wall-normal velocity profiles at (e) $R e_{\tau}=1000$, and (f) all $R e_{\tau}$. (a), (c), (e) Solid, dot-dashed and dashed lines indicate the global mean, zonal mean in the core and zonal mean outside of the core, respectively. Symbols are given in table 1. Open symbols indicate the zonal mean inside the core; closed symbols indicate the zonal mean outside the core.

of $\tilde{U}$ and $\hat{U}$, where the weights are determined by the intermittency, gives $\bar{U}$ according to

$$
\bar{U}=\gamma \hat{U}+(1-\gamma) \tilde{U}
$$

In figures $7(\mathrm{a})$ and (b), $\tilde{U}$ is only plotted for $z>0.1 \mathrm{~h}$ since the core very rarely drifts below the level $z=0.1 \mathrm{~h}$, as shown in figure $6(\mathrm{a})$. Consequently, $\bar{U}$ and $\hat{U}$ are almost identical for $z<0.1 h$. As expected, the mean streamwise velocity inside the core is 
approximately uniform. The mean velocity in the core scales with the centreline velocity, while the mean velocity profile outside the core does not $\left(U_{\tau}\right.$ and $\nu / U_{\tau}$ are the appropriate velocity and length scales in this turbulent region). Figures $7(\mathrm{c})$ and (d) show the zonal streamwise turbulent intensity profiles. $\widetilde{u^{2}}$ indicates the zonal mean streamwise turbulent intensity relative to $\tilde{U}$ (inside the core) and $\widehat{u^{2}}$ indicates the zonal turbulent intensity relative to $\hat{U}$ (outside the core), which are defined as

$$
\widetilde{u^{2}}=(\widetilde{U-\tilde{U}})^{2}, \quad \widehat{u^{2}}=(\widehat{U-\hat{U}})^{2} .
$$

The zonal turbulent intensities are to be interpreted differently from the conventional turbulent intensity since they are just a measure of variation in the streamwise velocity about the zonal mean in the respective region. The zonal mean turbulent intensity inside the core is considerably lower relative to that outside of the core for most of the channel. The fact that the zonal turbulent intensity outside the core is lower than that inside the core near the channel centreline indicates that, in the rare instances when the flow structures outside the core extend close to the channel centreline, the streamwise velocity of those structures does not deviate much from the zonal mean velocity, $\hat{U}$. The key point here is that the zonal turbulent intensity inside the core is low regardless of the wallnormal location of the core. These observations (zonal mean streamwise velocity is fairly uniform and the zonal mean turbulent intensity is low inside the core) provides further evidence that the core is a uniform momentum zone.

Figures $7(\mathrm{e})$ and (f) show the zonal mean wall-normal velocities, $\tilde{W}$ (inside the core), and $\hat{W}$ (outside). The mean wall-normal velocity is negative and positive inside and outside of the core, respectively. Inside the core, $\tilde{W}$ attains a minimum value of roughly -0.7 to $-0.9 U_{\tau}$ at $z \simeq 0.1 h$. Below this point $(z<0.1 h), \tilde{W}$ rapidly rises to zero. This explains the flat tops of the core boundary (observed in figure 5c), which result from the lateral expansion of the core caused by the sudden wall-normal deceleration as the core approaches the wall. Outside of the core, $\hat{W}$ peaks at $z \simeq 0.8 h$ with a fixed value of approximately $0.4 U_{\tau}$. The signs of $\tilde{W}$ and $\hat{W}$ indicate the core tends to move towards the wall, while the flow outside of it, possibly consisting entirely of flow structures originating from the wall, tends to move away from the wall. The implication is that wall-up motions take place below the core boundary, while 'top-down' motions (toward the wall) may be due to the core flow moving toward the wall (Hunt \& Morrison 2001). In fully developed channel flows, the downwards motion within the core is balanced with the upwards motion originating within the turbulent region (see figure 7e). In external flows (e.g. boundary layers), this is not the case and the downwards motion in the non-turbulent region is not as strong. This fundamental difference between channels and boundary layers is due to the existence of the opposing wall in the channel. The fully developed state of channel flows is reached when the shear layers originating from both walls are in balance and cannot grow any further. However, velocity fluctuations in these turbulent layers almost never directly interact with each other; they communicate via the quiescent core region. In constrast, as shown by Kim (1989), there is a direct wall-to-wall influence on the instantaneous pressure fluctuations across the channel, which can be one of the possible mechanisms responsible for the communication between two turbulent layers.

As shown in figure 4(b), the core can be discontinuous and this poses a problem for conditional analyses requiring continuous velocity traces. In all following analyses, only the instantaneous PIV vector fields where there is no discontinuity of the core are considered. Hence, $78.5 \%, 84.6 \%, 91.3 \%$ and $85.2 \%$ of the total PIV frames are considered in the analysis for datasets at $R e_{\tau}=1000,2000,3000$ and 4000, respectively. A second problem is that the wall-normal location of the velocity contour at $U=0.95 U_{C L}$ is 


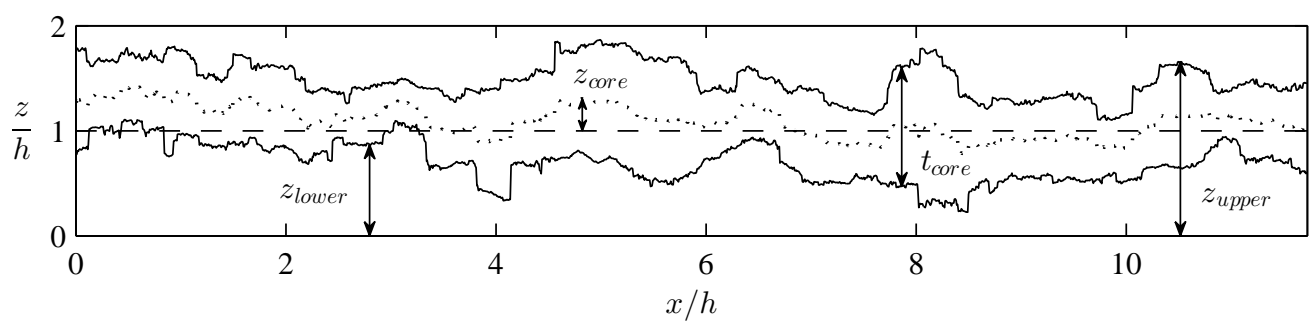

FIgURE 8. Parameters describing the core. The solid lines indicate the upper and lower enveloped boundaries of the core. The dotted line indicates the mid-point between $z_{\text {upper }}$ and $z_{\text {lower }}$. The dashed line indicates the channel centreline.
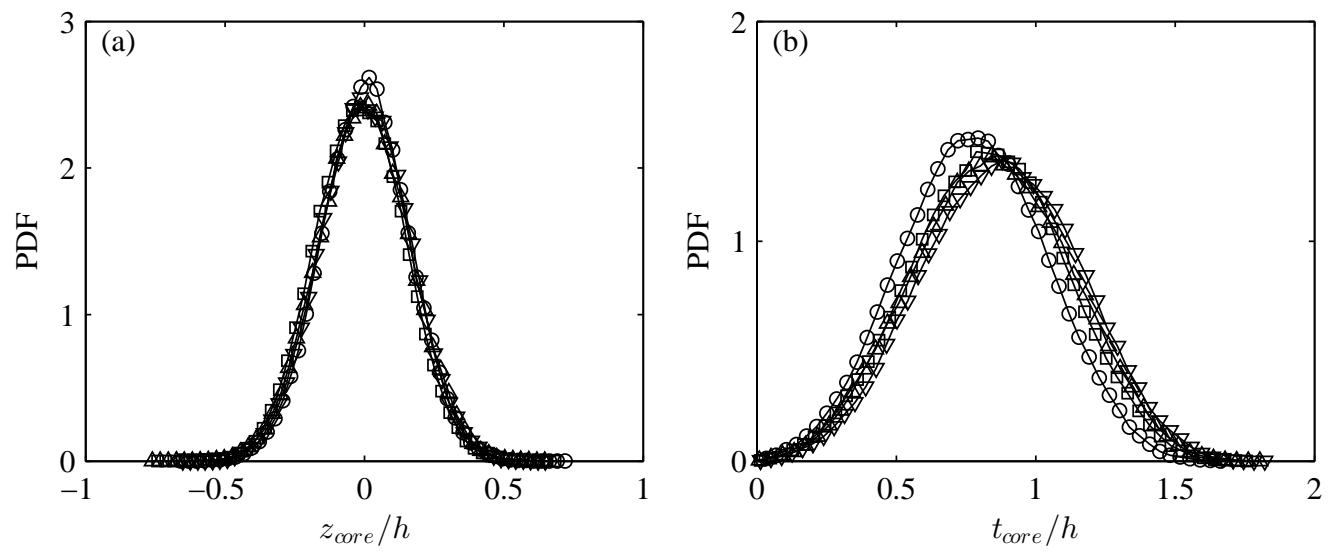

Figure 9. Distributions of (a) $z_{\text {core }}$ and (b) $t_{\text {core }}$ at $R e_{\tau}=1000,2000,3000$ and 4000. Symbols used are as in table 1.

a multivalued function due to the contour line folding back and forth (figure 4). To circumvent this problem, 'enveloped' core boundaries are used (e.g. Westerweel et al. 2002; Chauhan et al. 2014): The 'inner' enveloped core boundary takes the wall-normal position of the boundary that is furthest from the wall as shown in figure $6(\mathrm{~b})$. The core boundaries are 'inner' enveloped, rather than 'outer' (towards the wall) enveloped to ensure that no outside regions are included in the analysis of the enveloped core (although the choice of inner or outer enveloping does not change any conclusions drawn as shown in Appendix C). It is now possible to document statistics of the core and its boundary. The important parameters are illustrated in figure 8 and defined as:

- $z_{\text {upper }}$ and $z_{\text {lower }}$, which indicate the upper and lower core boundaries, respectively.

- $z_{\text {core }}$, which is the mid-point between $z_{\text {upper }}$ and $z_{\text {lower }}$ relative to the channel centreline and so represents the location of the centre of the core.

- The thickness of the core is denoted as $t_{\text {core }}=z_{\text {upper }}-z_{\text {lower }}$.

Figure 9(a) shows the PDF of the core location over the Reynolds number range. The distribution of $z_{c o r e}$ is Gaussian and does not show Reynolds number dependence. The mean value of $z_{\text {core }}$ is nominally zero (at the channel centreline), as the symmetry of the channel flow requires. $z_{\text {core }}$ mostly fluctuates within $\pm 0.5 h$ of the channel centreline, meaning the centre of the core often deviates a great distance from the centreline. Figure 9 (b) shows the PDF of the core thickness at various $R e_{\tau}$. The most notable observation is the large thickness, which takes an average close to $h$, such that on average half of the turbulent channel consists of approximately uniform momentum flow. Often the uniform 


\begin{tabular}{crrrrrrr}
\hline & \multicolumn{2}{c}{$z_{\text {core }}$} & \multicolumn{2}{c}{$t_{\text {core }}$} & \multicolumn{2}{c}{$z_{\text {lower }}$} \\
& \multicolumn{1}{c}{$\mu / h$} & $\sigma / h$ & $\mu / \mathrm{h}$ & $\sigma / \mathrm{h}$ & $\mu / \mathrm{h}$ & $\sigma / \mathrm{h}$ \\
& & & & & & \\
\hline $1030(\approx 1000)$ & 0.004 & 0.156 & 0.778 & 0.260 & 0.611 & 0.203 \\
$2100(\approx 2000)$ & -0.014 & 0.160 & 0.830 & 0.272 & 0.585 & 0.210 \\
$3090(\approx 3000)$ & -0.001 & 0.163 & 0.846 & 0.278 & 0.577 & 0.214 \\
$3965(\approx 4000)$ & 0.002 & 0.159 & 0.876 & 0.280 & 0.562 & 0.212 \\
\hline
\end{tabular}

TABLE 2. The mean, $\mu$, and the standard deviation, $\sigma$, of inner-enveloped core variables: $z_{\text {core }}$, $t_{\text {core }}$ and $z_{\text {lower }}$ as fractions of the channel half height, $h$.

momentum core can occupy $3 / 4$ of the channel. The distribution of $t_{\text {core }}$ also follows a Gaussian distribution, however, the mean core thickness increases with increasing $R e_{\tau}$. Table 2 summarizes the mean value, $\mu$, and the standard deviation, $\sigma$, of $z_{\text {core }}$ and $t_{\text {core }}$. The average thickness of the inner enveloped core ranges from $0.78-0.88 h$ for $R e_{\tau}=1000-4000$, respectively, with an increasing trend as $R e_{\tau}$ increases (Note that the average thickness of the 'outer' enveloped core is $0.98 h$ at $R e_{\tau}=4000$ - see Appendix C). This explains why the flow spends more time in the core at a given wall-normal location at higher $R e_{\tau}$, as shown by the intermittency profiles in figure 6 . However, it is not yet clear what the asymptotic limit of the core thickness will be at infinite Reynolds number. Table 2 also shows the mean and standard deviation of $z_{\text {lower }}$, describing the distribution of the distance from the wall to the edge of the core. The mean value of $z_{\text {lower }}$ is simply the half of area outside of the core, as expected. Figure 10(a) shows the joint PDF between $t_{\text {core }}$ and $z_{\text {core }}$ at $R e_{\tau}=1000$ (only one Reynolds number is shown for clarity; the joint PDF is similar at all other $\left.R e_{\tau}\right)$. When $z_{\text {core }}$ is near the channel centreline, the thickness of the core varies across the full range of $t_{\text {core }}$, but when the core is near the wall, its thickness is limited by the existence of the wall. In other words, when the core is very thick, it must be located closer to the channel centreline; however, when the core is thin, it can be located over a greater range of distances from the centreline.

\subsection{Large scale meandering of the core}

The channel core exhibits thickening and thinning (variation in the thickness) and meandering behaviour (variation in the position). Examples of these behaviours can be seen in figure 4 and 5 . In order to determine the dominant length scale of the core, pre-multiplied spectra of $t_{\text {core }}$ and $z_{\text {core }}$ are plotted in figure $10(\mathrm{~b})$. In this figure $\phi_{z z}, \phi_{t t}$ are the power spectral densities of $z_{\text {core }}$ and $t_{\text {core }}$ respectively, $k_{x}$ is the streamwise wavenumber and $\lambda_{x}=2 \pi / k_{x}$ is the streamwise wavelength. Both spectra for $t_{\text {core }}$ and $z_{\text {core }}$ reach a maximum at a wavelength of about $0.2 \mathrm{~m}=4 h$ in the streamwise direction. The core location arguably peaks at $\lambda_{x}=4 h$, which is of a similar value to the characteristic wavelength (3.2h) of anti-symmetric flow structures in channel flow reported by Teitel \& Antonia (1990). This raises the question of whether the flow is dominantly antisymmetric.

The channel core can be categorised into symmetric and anti-symmetric modes by analysing the positions of the core boundary. The core will be classified as symmetric when the the fluctuations of $z_{\text {upper }}$ and $z_{\text {lower }}$ are negatively correlated; that is, when

$$
S_{y}=\left(z_{\text {upper }}-\overline{z_{\text {upper }}}\right)\left(z_{\text {lower }}-\overline{z_{\text {lower }}}\right)
$$

is negative on average over the selected velocity field, the core in that field is symmet- 

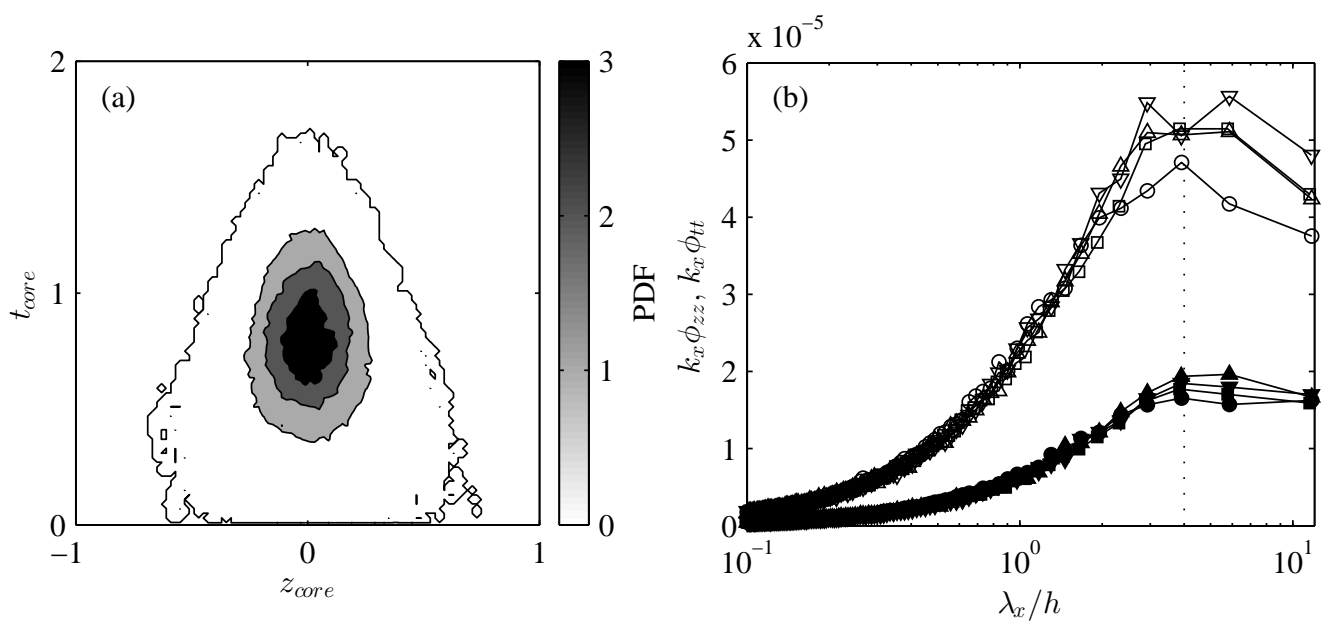

FiguRE 10. (a) joint PDF between $t_{\text {core }}$ and $z_{\text {core }}$ at $R e_{\tau}=1000$. (b) Pre-multiplied spectra of the thickness of the core and the position of the core at all $R e_{\tau}$. The open symbols indicate the spectra of $t_{\text {core }}$ and the closed symbols indicate the spectra of $z_{\text {core }}$. The vertical dotted line indicates $\lambda_{x} / h=4$. Symbols used are as in table 1 .

ric. The core is antisymmetric when $S_{y}>0$ on average. Figure 11(a) shows an example of a symmetric core overlaid on the instantaneous streamwise velocity field. Here, one can observe that the core is dominantly symmetric about the channel centreline. The fluctuating streamwise velocity field from the same PIV frame (figure 11b) reveals that the symmetric core is associated with large-scale symmetric flow structures. Similarly, the core is classified as anti-symmetric when the the fluctuations of $z_{\text {upper }}$ and $z_{\text {lower }}$ are positively correlated. Figures 11 (c) and (d) show an example of the anti-symmetric core along with the corresponding fluctuating streamwise velocity field. In contrast to the symmetric core example, the anti-symmetric core is associated with large-scale antisymmetric flow structures. Approximately $70 \%$ of the PIV frames are dominated by an anti-symmetric core, independent of Reynolds number, indicating that the core of the channel flow is more often anti-symmetric than symmetric. This anti-symmetric nature of the core and the associated large-scale anti-symmetric instantaneous streamwise fluctuating velocity fields are consistent with the anti-symmetry of the large-scale structures in channel flows reported by Teitel \& Antonia (1990) and Jiménez et al. (2010).

The visualisations in figure 11 as well as the dominant anti-symmetric modes suggest that the large wavelengths of the core might be associated with an 'inviscid' instability mechanism commonly encountered in free-shear flows, such as mixing-layers, jets and wakes. In fact, the visual similarity of the core boundary and the Kelvin-Helmholtz instability of jets was part of the motivation of this study. Furthermore, figure 5 suggests the core most likely also undergoes a spanwise secondary instability, with similarities to the 'braid' region of a mixing layer (Bernal \& Roshko 1986). The noted spanwise wavelength of approximately $h$ is indicative of such a mechanism.

Due to the large streamwise extent of the experimental field of view, the core often displays both symmetric and anti-symmetric modes within a single field. Therefore, in the following core symmetry analysis we only consider instantaneous PIV fields where the magnitude of covariance of $z_{\text {upper }}$ and $z_{\text {lower }}$ is the top $50 \%$. Based on these fields, figure 12(a) shows the distribution of $z_{\text {core }}$ when the core is symmetric and anti-symmetric (only one Reynolds number is shown in the figure for clarity; no significant Reynolds 

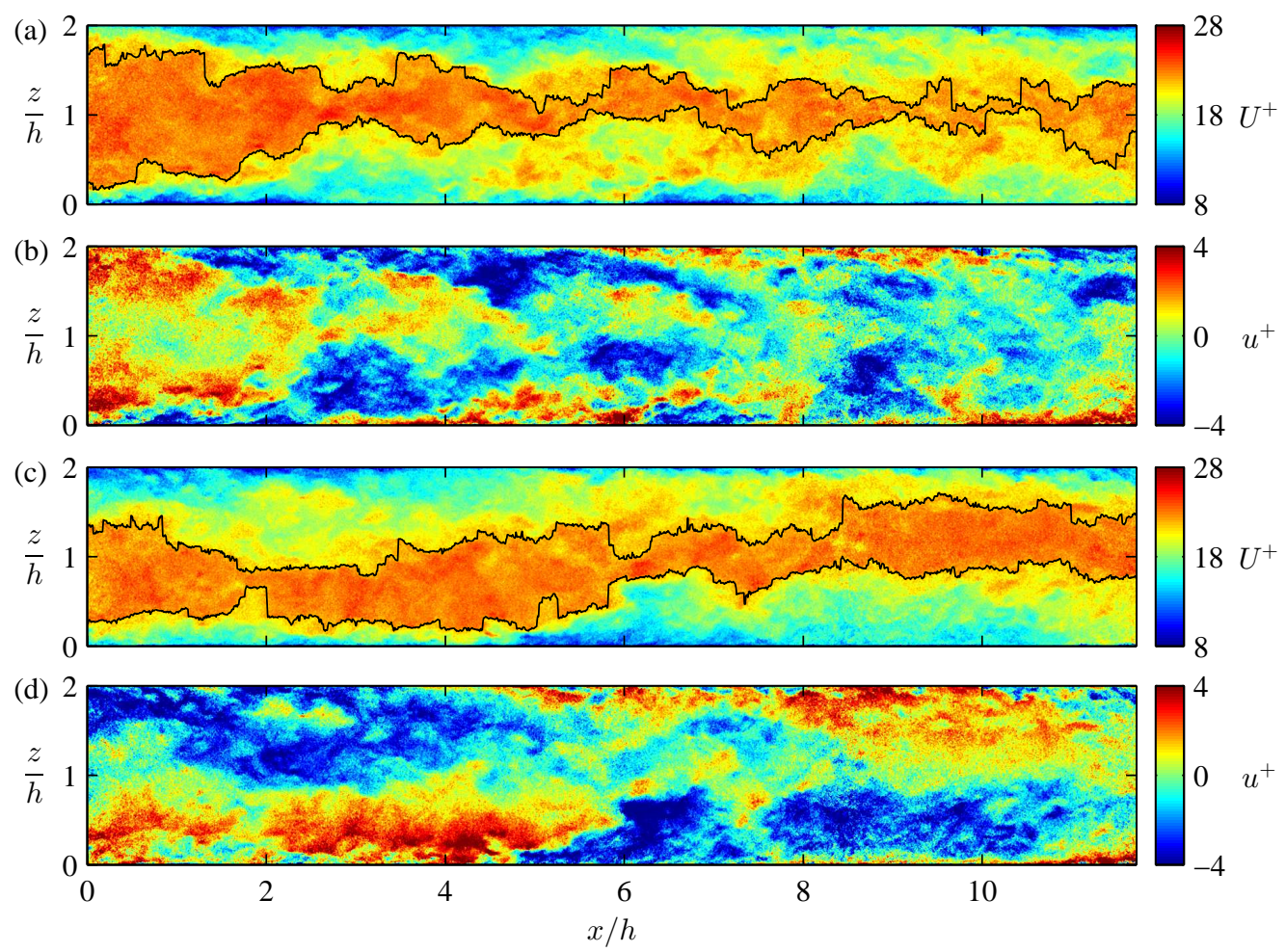

Figure 11. (a) Example of a symmetric core along with (b) the corresponding $u^{+}$field. (c) Example of an anti-symmetric core along with (d) the corresponding $u^{+}$field. Black lines in (a) and (c) indicate the enveloped boundaries of the channel core.
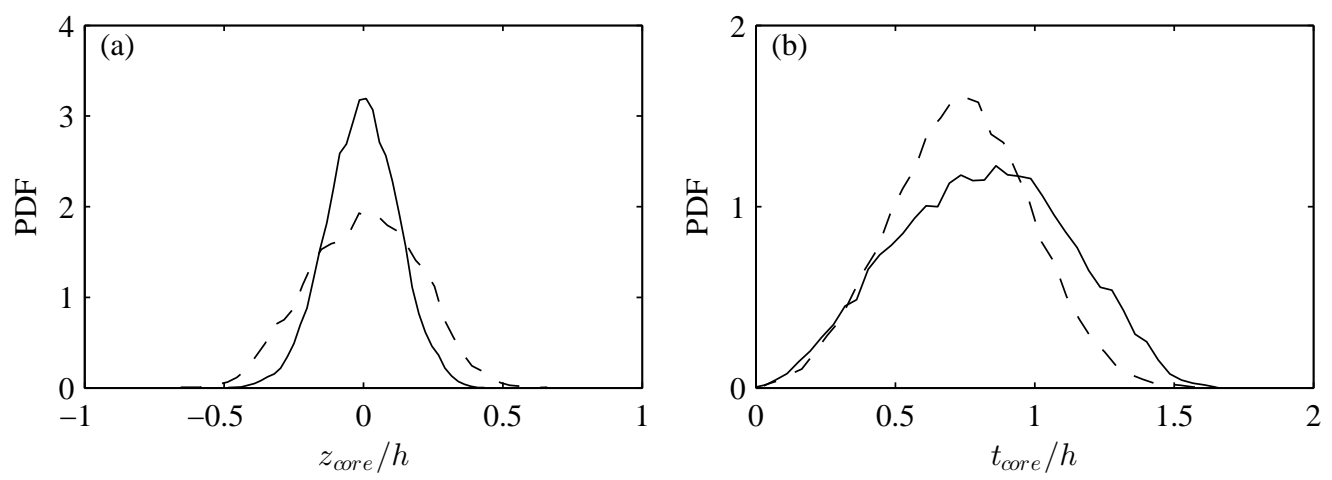

FIgURE 12. Distributions of (a) $z_{\text {core }}$ and (b) $t_{\text {core }}$ for symmetric (solid lines) and anti-symmetric (dashed lines) core at $R e_{\tau}=1000$.

number trends were found). The PDF shows that the core oscillates much more about the channel centerline when the core is anti-symmetric (the standard deviation of the core location is $50 \%$ higher for anti-symmetric cores). Figure 12(b) shows the core is thicker on average and the thickness has more variation when the core is symmetric. The overall picture is one where the most common anti-symmetric core is thinner and oscillates about the centreline more than the symmetric core. Even so, the thickness of the core is mostly a large fraction of the channel height. 


\subsection{Summary}

By defining a core as the highest uniform momentum zone in channel flow, it is possible to investigate the contribution of regions of the flow with small variation in velocity. The large size of the core and the amount of time the flow spends inside this core was somewhat surprising and indicates that the channel flow, like external boundary layers, entrains large regions of relatively uniform momentum. Whilst it is generally thought that internal flows, such as channels, consist mostly of highly turbulent fluid, the results here suggest a different view where beyond $z \approx 0.55 h$ the flow spends more than $50 \%$ in the uniform momentum core. Even as close as $z / h \approx 0.3$ there is a significant fraction of the flow that is inside this core region $(\sim 10 \%)$. The uniform momentum core is mostly anti-symmetric, similar to the interface of jets and wakes, and occupies nearly half of the channel on average.

\section{Flow properties near the core boundary}

Conditional averages are computed across the core boundary to determine the relative turbulence activity in the core region. Figure 13(a) shows the conditional average of streamwise velocity. The quantities enclosed by angle brackets, \langle\rangle , indicate quantities that are conditionally averaged across the core boundary. $\zeta$ indicates the coordinate attached at the core boundary, that is parallel to the wall-normal direction, pointing towards the interior of the core. Hence, the region with $\zeta>0$ indicates the core. A sharp jump in velocity is evident as the core boundary is crossed (coming from the wall). This jump is consistent with the PDF shown in figure 3(f) which shows a sharp increase in probability for velocities above $0.95 U_{C L}$. The actual values of the jump in velocity, $\mathcal{D}[\langle U\rangle]$ is about $0.06 U_{C L}$, with no clear Reynolds number trend. The velocity jump is defined in the manner of Chauhan et al. (2014). In figure 13(b) the conditional averages of spanwise vorticity, $\omega_{y}$, are shown, illustrating that the $0.95 U_{C L}$ threshold passes through a region of strong positive vorticity (so-called 'prograde' vorticity). The peak vorticity at the core boundary is much larger than the local mean vorticity as expected due to the high shear (strong velocity gradient seen in figure 13a) across the core boundary. Following Brown \& Roshko (1974) and Chauhan et al. (2014) a vorticity thickness, $\delta_{\omega}$, is defined as

$$
\delta_{\omega} \equiv \frac{\mathcal{D}[\langle U\rangle]}{\left.\frac{d\langle U\rangle}{d z}\right|_{\max }} .
$$

It is found that $\delta_{\omega}=0.015 h$, which represents the thickness of the shear layer along the core boundary where concentrated vorticity causes the jump in streamwise velocity.

To examine the relative turbulence activity in the core as compared to the outside of the channel, the total velocity is decomposed in the frame of reference of the core boundary, in the same manner that it was decomposed in Bisset et al. (2002), as shown in equation 5.2 .

$$
u^{*}(x, \zeta)=U(x, \zeta)-\langle U(x, \zeta)\rangle
$$

Here, $\langle U(x, \zeta)\rangle$ indicates the conditional average of $U$ across the core boundary (figure 13a) and $u^{*}$ indicate the instantaneous fluctuation of $U$ with respect to the conditionally averaged profile of $\langle U\rangle$. The same decomposition is applied to the wall-normal velocity $W$. The conventional Reynolds decomposition of the total velocity does not give the true measure of fluctuating velocity components inside the core since the actual mean velocity in the core can be different to the conventional time-averaged velocity as the core oscillates up and down in the channel. 

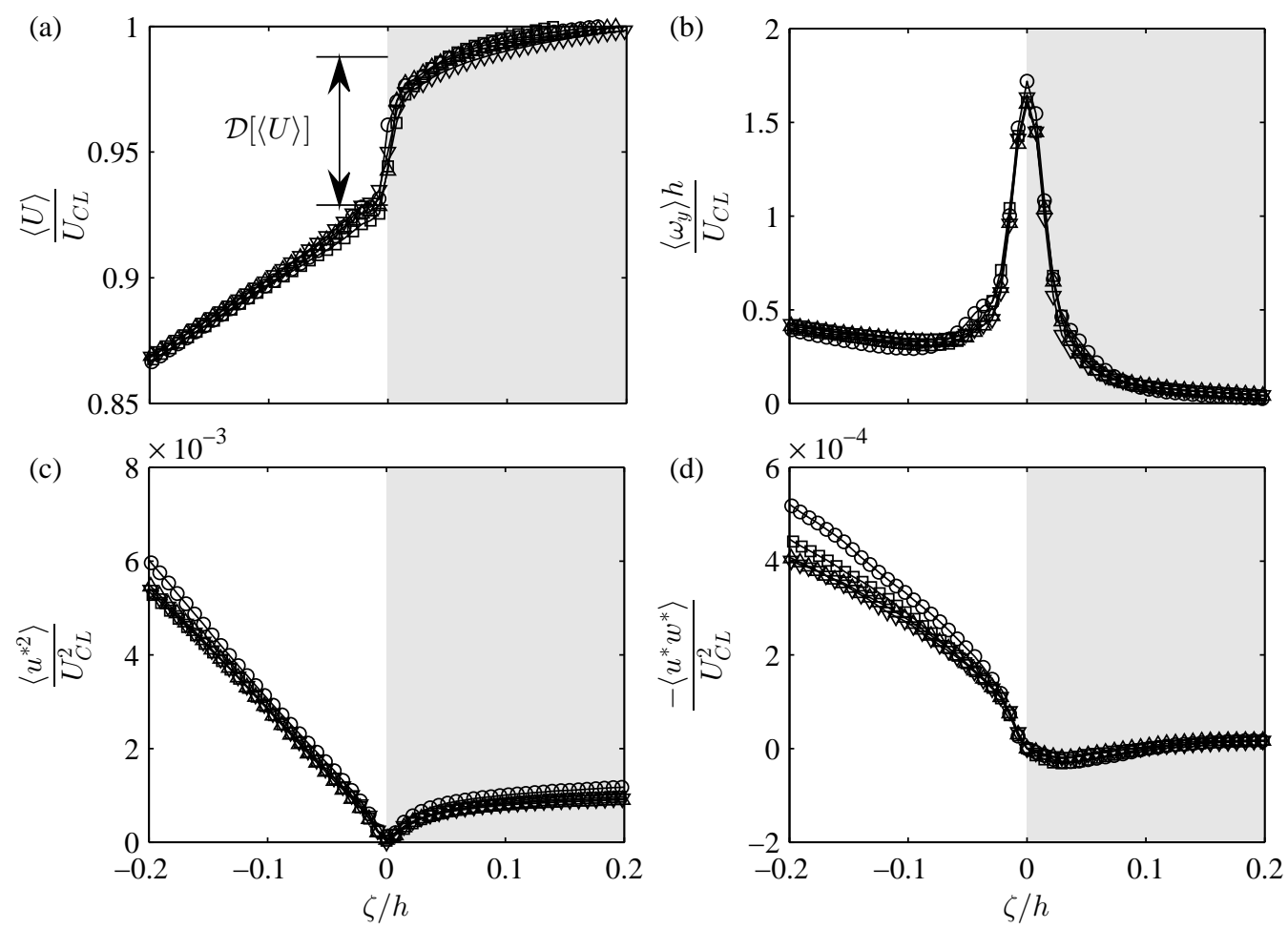

FIGURE 13. Conditional average of (a) Streamwise velocity (b) Spanwise vorticity (c) turbulent kinetic energy and (d) shear stress across the core boundary as a function of $\zeta$, normalized with $h$ and $U_{C L}$. Symbols used are as in table 1. Gray shading highlights the core region.

Figure 13(c) displays the conditional streamwise turbulence intensity in the reference frame of the core boundary, $\left\langle u^{* 2}\right\rangle$. The local minimum at the core boundary $(\zeta=0)$ is a manifestation of the core boundary being defined by a contour line of fixed streamwise velocity $\left(U=0.95 U_{C L}\right)$. In other words, the streamwise velocity fluctuation along the core boundary is fixed $\left(u^{*}=0\right)$. Figure 13(c) shows that the value of $\left\langle u^{* 2}\right\rangle$ drops to a small value across the core boundary and remains low in the core as compared with the flow outside of the core. The conditional turbulent intensity of the core is about $3 \%$ of $U_{C L}$ through the majority of the core, which occupy almost half of the channel (slightly less than the unconditional turbulence intensity of $3.8 \%$ of $U_{C L}$ at the channel centreline). So although the streamwise turbulence intensity inside the core is low, the core flow is still undoubtedly turbulent. This represents the well-known fundamental difference between channels and boundary layers: the core is weakly turbulent while the free stream outside a boundary layer is completely non-turbulent. Even so, there are clearly analogous behaviours between TNT interfaces and the core boundary.

Figure 13(d) shows the conditional shear stress $\left\langle u^{*} w^{*}\right\rangle$. This represents the wall-normal transport of streamwise velocity in the reference frame of the core boundary, which is comparable to the Reynolds shear stress in the conventional reference frame. When crossing the core boundary, the value of $\left\langle u^{*} w^{*}\right\rangle$ drops to almost zero and remains very small inside of the core. No differences in the conditionally averaged statistics when the core is symmetric and anti-symmetric are observed (not shown in this paper). This suggests that, although the large-scale influence of the opposing wall can cause the core 

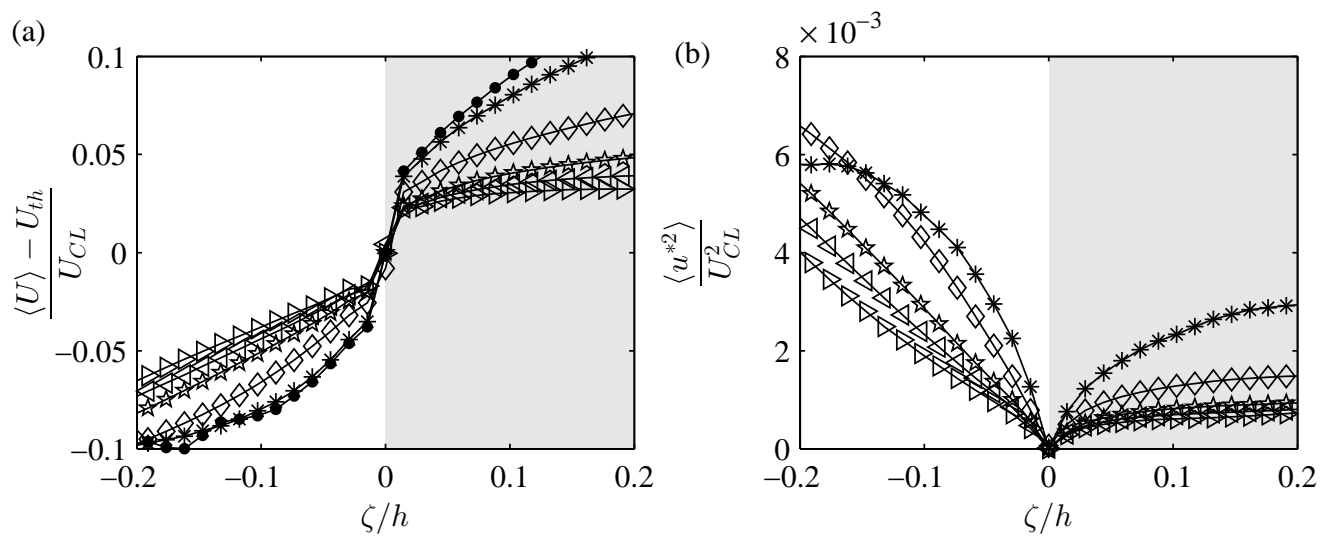

FiguRE 14. Conditional averages of (a) streamwise velocity and (b) turbulent kinetic energy, $\left\langle u^{* 2}\right\rangle$, across the various streamwise velocity contours as a function of $\zeta$ at $R e_{\tau}=4000$. Symbols represent different threshold levels of $\bullet: 0.75 U_{C L}, *: 0.8 U_{C L}, \diamond: 0.9 U_{C L}$, 祘: $0.95 U_{C L}, \triangleleft: 0.97 U_{C L}$, $\triangleright: 0.99 U_{C L}$. Gray shading highlights the core.

to be symmetric or anti-symmetic, the small-scale flow structures near the core boundary are unaffected.

Figure 13 serves to show that there is a core of relatively high and uniform velocity, low vorticity, low turbulence intensity and low transport of streamwise momentum in channel flow. These properties suggest that the flow inside the core is 'quiescent'. As discussed previously, this 'quiescent core' can often occupy a large percentage of the channel cross-section.

In this investigation, our core identification scheme is based on a threshold of streamwise velocity and some similarities are apparent between TNT interfaces and the core boundary. It is perhaps worth noting that, when compared with the vorticity based scheme used for detection of TNT interfaces, good qualitative agreement between this and our velocity threshold method is observed (see Appendix B).

\section{A note on uniform momentum zones}

In section 3, the technique used to identify the core was based on the notion of uniform momentum zones. Instantaneously, the core is not the only uniform momentum zone as indicated by the PDFs of streamwise velocity in figure 3(b, c). In fact, Meinhart \& Adrian (1995) have shown that the turbulent flow in the outer region consists of uniform momentum zones separated by shear layers with sharp gradients in velocity and marked changes in other turbulence quantities. Statistically then, in relation to the preceding discussion, this flow structure will result in a jump in conditionally averaged velocity across a boundary defined by any velocity contour. This is illustrated in figure 14(a) where conditionally averaged velocity profiles are shown for a range of velocity thresholds. Figures 15 shows instantaneous snapshots of $U^{+}$and the corresponding $d U^{+} / d z^{+}$fields with streamwise velocity contour lines ranging from $0.65 U_{C L}$ to $0.95 U_{C L}$. Between adjacent local uniform momentum zones there are regions of high shear (black coloured regions in figure 15b) where velocity contour lines bunch together and a sharp jump in velocity in the wall-normal direction occurs. Although the turbulence consists of many uniform momentum zones, they do not have statistically preferred streamwise momentum values, except for the core, as shown in figure $3(\mathrm{f})$.

So, while there are many uniform momentum zones (defined by arbitrary velocity 
(a)

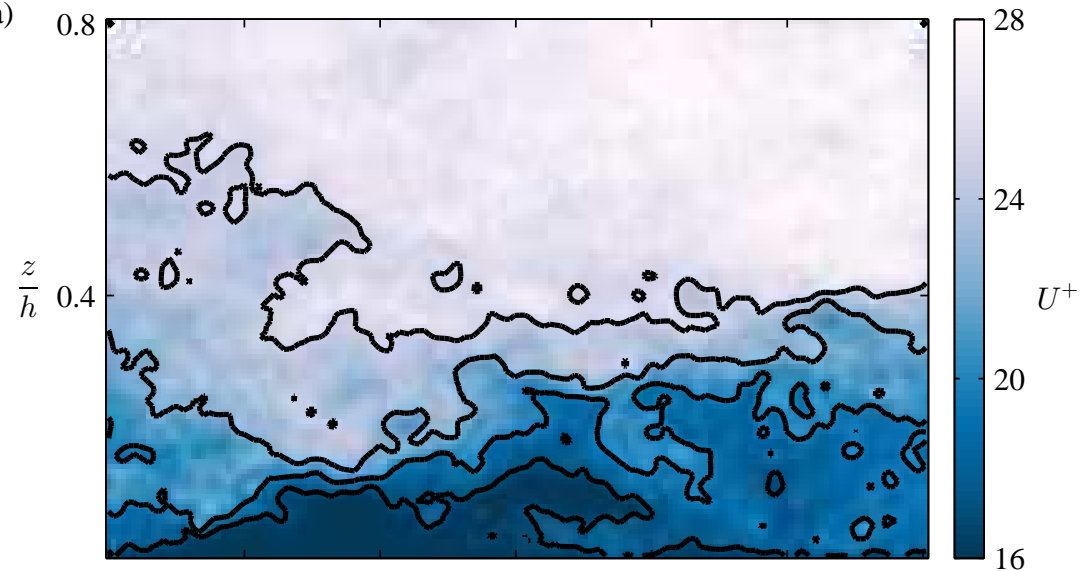

(b)

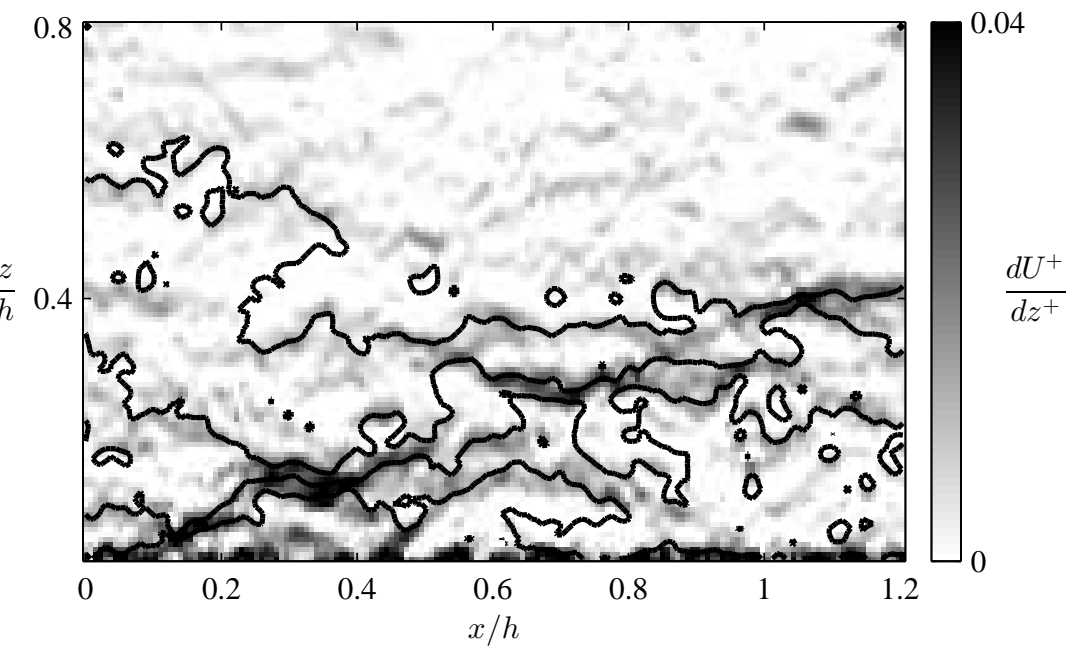

Figure 15. Example contour plots of instantaneous (a) $U^{+}$and (b) $d U^{+} / d z^{+}$at $R e_{\tau}=4000$. Black lines indicate contours of $U$ from $0.65 U_{C L}$ to $0.95 U_{C L}$ with increments of $0.1 U_{C L}$.

thresholds), the core is unique. Further evidence for this is provided in figure 14(b), where profiles of $\left\langle u^{* 2}\right\rangle$ across a range of streamwise velocity contours are plotted. When the threshold defining the zone boundary is greater than $0.95 U_{C L}$, the values of $\left\langle u^{* 2}\right\rangle$ inside of the boundary are independent of the threshold, indicating that the regions bounded by the zone boundaries with the threshold greater than $0.95 U_{C L}$ are in the same uniform momentum zone, defined as the 'core'. Yet there is a strong dependence on threshold when the threshold is less than $0.95 U_{C L}$. This confirms the earlier observation that the velocity contour $0.95 U_{C L}$ defines the boundary of the highest uniform momentum zone, which we call the 'core'. The core is also the uniform momentum zone with the minimum turbulence level.

\section{Conclusions}

From a large-scale PIV experiment in turbulent channel flow, a zone of uniformly high momentum was identified by examination of the PDF of streamwise velocity by extending the method of modal velocity originally developed by Adrian et al. (2000). This uniform 
momentum zone which resides at the centre of channel is termed the 'core' region. The PDF analysis culminated in a core boundary quite simply defined by a velocity contour, or threshold, of $U=0.95 U_{C L}$. Conditional averages across the core boundary show that the core is a region with low turbulence and momentum transport levels. Consequently, we call the core a 'quiescent core'. This suggests that the core is, in some respects, analogous to the free-stream in a boundary layer. There is also a jump in streamwise velocity and a peak in vorticity due to high shear across the core boundary; features which have also been documented at the turbulent/non-turbulent interface of boundary layers (and jets and wakes, see Kovasznay et al. 1970; Westerweel et al. 2002; Chauhan et al. 2014).

The core is quite thick, spanning about $40-45 \%$ of the channel, on average, for the range of Reynolds numbers considered here. The intermittency, defined in a similar way as in external flows, shows that the low turbulence core dominates the flow for $z>0.55 h$ and even penetrates as low as $z \approx 0.2 h$ for significant time periods.

Although turbulent channel flow is commonly thought of as being fully turbulent, with the implication that there is no identifiable boundary between high and low turbulence regions, the findings of this work suggest a somewhat alternative view of channel flow. Qualitatively, when viewed from one wall upwards, turbulence is produced and dissipates with distance from the wall while entraining and mixing low turbulence fluid from the core, similar to boundary layers except that boundary layers entrain precisely nonturbulent fluid (ideally). In this sense, internal flows may be more similar to boundary layers than previously thought (although there are documented quantitative differences Monty et al. 2009; Jiménez et al. 2010). This follows the work of Dean \& Bradshaw (1976) who posited that channel flow could be thought of as two interacting shear layers from each wall, although they made no mention of a low turbulence region separating them. Despite these apparent similarities, we note that instantaneously flow structures attached to one channel wall can be influenced by those on the opposing wall. This leads to anti-symmetric behaviour of the core. Such interactions are absent in external flows, and hence the analogy between boundary layers and channels is limited.

Finally, a symmetry analysis of the core shows that a symmetric or anti-symmetric core coincides with large-scale symmetric or anti-symmetric velocity fields closer to the wall, respectively. The core is most commonly anti-symmetric and oscillates about the channel centreline, with long wavelengths $\lambda_{x}>4 h$ in the streamwise direction. The predominant anti-symmetric nature of these modes, combined with the quiescence of the core suggests that the characteristic wavelength maybe due to an instability mechanism similar to that in free-shear flows, encouraging further work in this area.

The authors wish to acknowledge the financial support of the Australian Research Council and the Defence Science and Technology Organisation. The authors are grateful to Prof. J. Jimenez, Prof. R. D. Moser, Dr. J. C. del Alamo and Dr. P. S. Zandonade for making the channel DNS data available.

\section{Appendix A. Validation of the first and second order statistics}

To validate the accuracy of the experimental results obtained from the planar PIV datasets, mean velocity and turbulent intensity profiles are presented in figure 16 . Here we present data from the four Reynolds numbers acquired, together with equivalent DNS and hot-wire anemometry data at available Reynolds numbers. The hot-wire data has a spatial averaging of 22 viscous length scales in the spanwise direction and comes from measurements in the same facility ( $\mathrm{Ng} 2011$ ). Good agreement is observed for the 

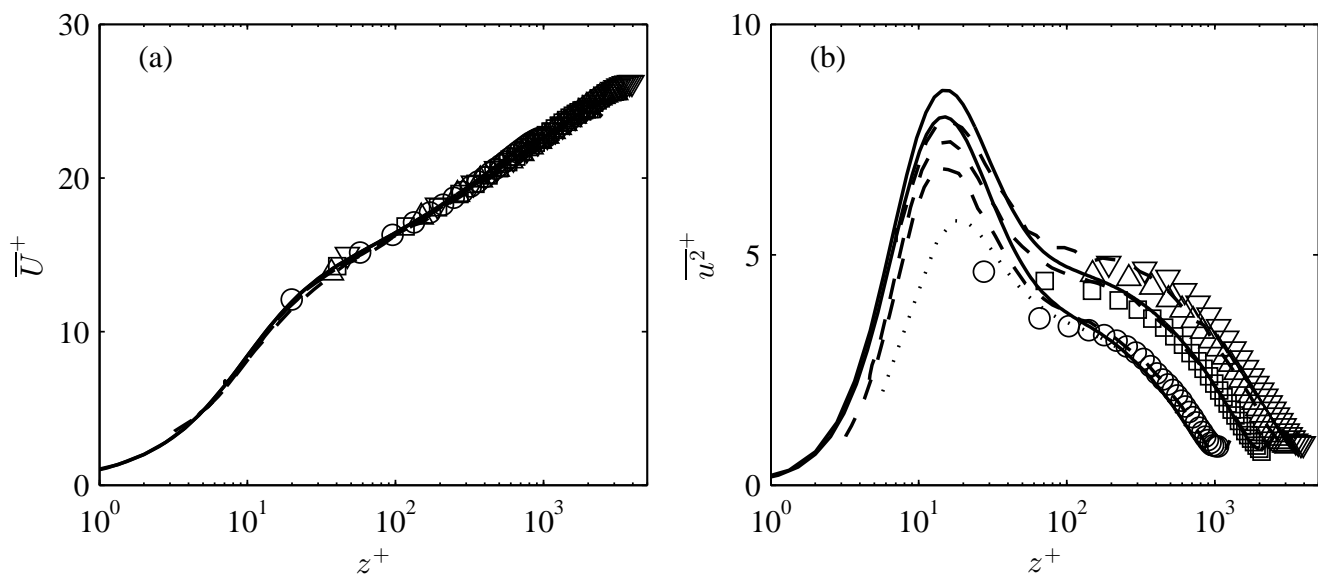

FIGURE 16. Comparison of the flow statistics between experimental results and DNS results (a) The mean streamwise velocity, $\bar{U}^{+}$and (b) The turbulence intensity, ${\overline{u^{2}}}^{+}$. The solid lines indicate the statistics for DNS data. The dashed lines indicate the statistics for hot-wire data. The dotted line indicates the filtered DNS data. Symbols indicate PIV data (see table 1).

streamwise mean velocity. Note that, although data in the near wall region was captured $\left(z<1.5 \mathrm{~mm}\right.$, or $z^{+}<30$ at $\left.R e_{\tau}=1000\right)$, reliable vector fields were not obtainable in this region due to issues such as reflections. Therefore, these data are not included in any preceding analyses.

The streamwise turbulence intensity (shown in figure 16b) indicates that the planar PIV measurements are able to resolve the structure further from the wall, which is sufficient for the aims of this study. Spatial attenuation is observed in the near wall region, which is dominated by the small scales present in the flow. This is expected due to the spatial averaging caused by the interrogation window size. By taking DNS data and simulating the spatial averaging with a box-filter of length 15 viscous units in the streamwise and wall-normal directions and 30 viscous units (accounting for the laser sheet thickness of approximately $1.5 \mathrm{~mm}$ ) in the spanwise direction, it is confirmed that discrepancies between the PIV and DNS turbulence intensities are due to this issue. An example is shown in figure 16 (b) for $R e_{\tau}=1000$ (dotted line).

\section{Appendix B. Validation of the core detection method}

In order to validate the detection method of the core, the detected core is compared with vorticity criteria, which is commonly used to detect interfaces. Here, we define the non-dimensional enstrophy, $\mathcal{E}^{+}$, as a measure of the instantaneous vorticity magnitude.

$$
\mathcal{E}^{+}=\frac{\nu^{2}}{2 U_{\tau}^{4}}\left(\omega_{x}^{2}+\omega_{y}^{2}+\omega_{z}^{2}\right)
$$

Figure 17 shows the core boundary $\left(0.95 U_{C L}\right.$ streamwise velocity contour) on top of the enstrophy spots where $\mathcal{E}^{+}$is greater than 0.007 using DNS data. The cut-off value for $\mathcal{E}^{+}$is chosen arbitrarily since we are only making a comparison. Although not being completely non-turbulent, the population density of enstrophy spots (where $\mathcal{E}^{+}>0.007$ ) inside the core is clearly much lower than that outside of the core, demonstrating that the core boundary demarcates the regions with high and low enstrophy. In channel flow, the enstrophy spots exist in patches and do not fill the entire area outside of the core as indicated by white areas outside of the core in figure 17. However, the majority of area 


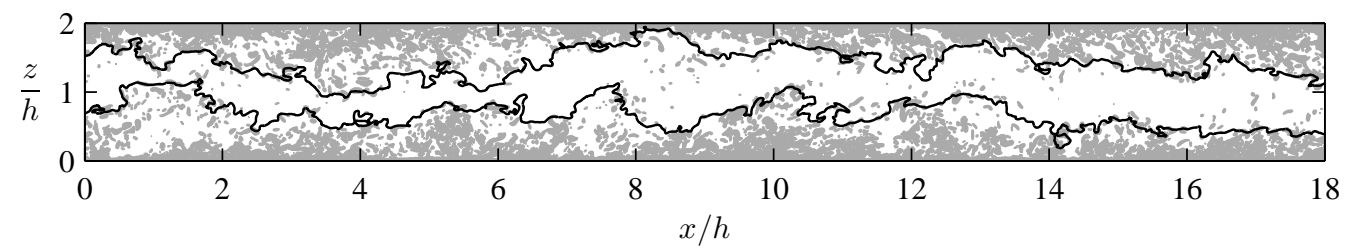

Figure 17. Plot of the detected core boundary on top of enstrophy spots $\left(\mathcal{E}^{+}>0.007\right)$. Black solid lines indicate the core boundaries and gray filled areas indicate enstrophy spots.

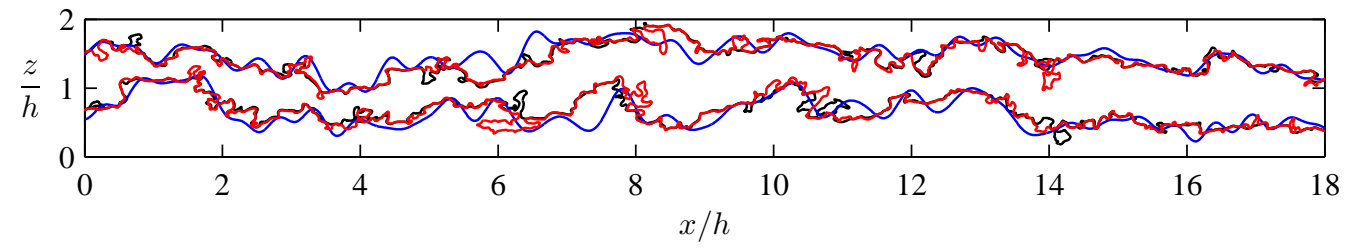

Figure 18. Comparison of boundaries based on various quantities. Black, blue and red lines indicate the core boundary $\left(U=0.95 U_{C L}\right.$ contour $)$, the filtered enveloping lines of enstrophy spots $\left(\mathcal{E}^{+}>0.007\right)$ and isocontour lines of $k=0.4$, respectively.

outside the core is still occupied with densely populated enstrophy spots. Therefore, it does not affect the conclusion drawn in this paper that the flow inside the core is more quiet relative to the flow outside the core regardless of the instantaneous position and thickness of the core.

In PIV datasets, spatial averaging effect and experimental noise would make any vorticity based detection scheme unreliable. However, in DNS datasets, enstrophy based scheme could also be employed to detect the core, if the choice of threshold can be justified and the difficulties in tracking the core boundary, caused by the patchiness of the enstrophy spots, can be resolved.

For a more comprehensive comparison between different identification methods, the boundaries determined based on $k$ and $\mathcal{E}^{+}$are plotted alongside our core boundary based on $U$ in figure 18 . Here, $k$ represents the turbulence detector function employed by Chauhan et al. (2014). For channel flow, $k$ is defined to be the mean value of squared fluctuation about the mean centreline velocity in a $3 \times 3$ grid as shown below.

$$
k=100 \times \frac{1}{9 U_{C L}^{2}} \sum_{m, n=-1}^{1}\left[\left(U_{m, n}-U_{C L}\right)^{2}+\left(W_{m, n}\right)^{2}\right]
$$

For $k$, isocontour lines of $k=0.4$ are used as the boundary (c.f. threshold of $k=$ 0.12 used in boundary layer by Chauhan et al. (2014)). For $\mathcal{E}^{+}$, lines enveloping the furthest enstrophy spots from each respective wall are first determined. Due to patchiness of the enstrophy spots, the enveloping lines are spiky. In order to remove the spikes, the enveloping lines are passed through a low-pass filter that removes signals with a wavelength less than $0.5 h$. This cut-off wavelength is approximately five times smaller than the typical length scale $(2-3 h)$ of large scale motions (LSMs) in wall-bounded turbulence so the filtered enveloping lines correctly represent the large-scale behaviour of the boundary based on $\mathcal{E}^{+}$. We note that this is one way of dealing with patchiness of the enstrophy spots, but not the only possible way of doing so. Figure 18 shows a good agreement between all three boundaries. Although the threshold values for $k$ and $\mathcal{E}^{+}$are arbitrarily chosen, it demonstrates that the core can also be detected by means of quantities other than $U$ with an appropriate choice of threshold, which will depend on 
(a)

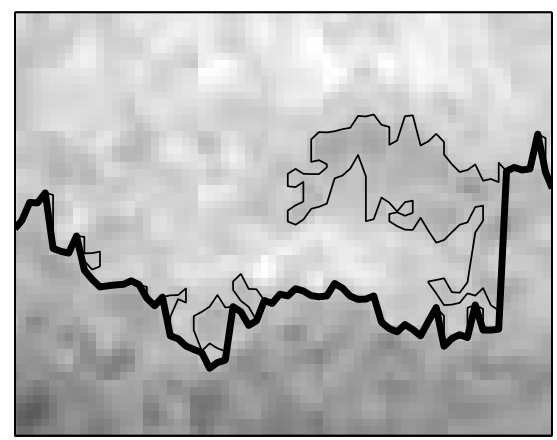

(b)

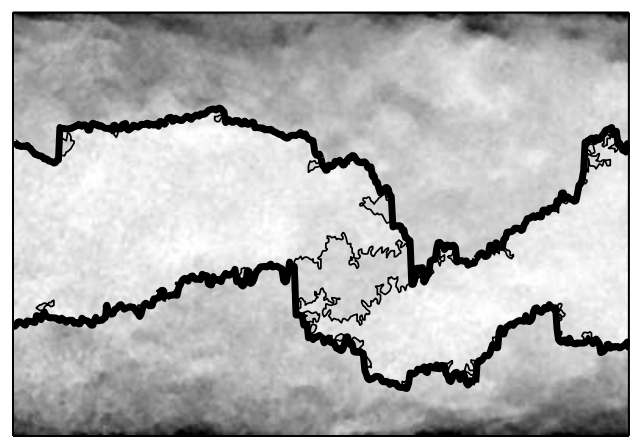

Figure 19. (a) Example of an 'outer' enveloped upper core boundary. (b) Example of an 'outer' enveloped core boundary when two disconnected (in the streamwise-wall-normal plane) cores overlap. The thin line indicates un-enveloped core boundary (contour line of $0.95 U_{C L}$ ). The thick line indicates the 'outer' enveloped core boundary.
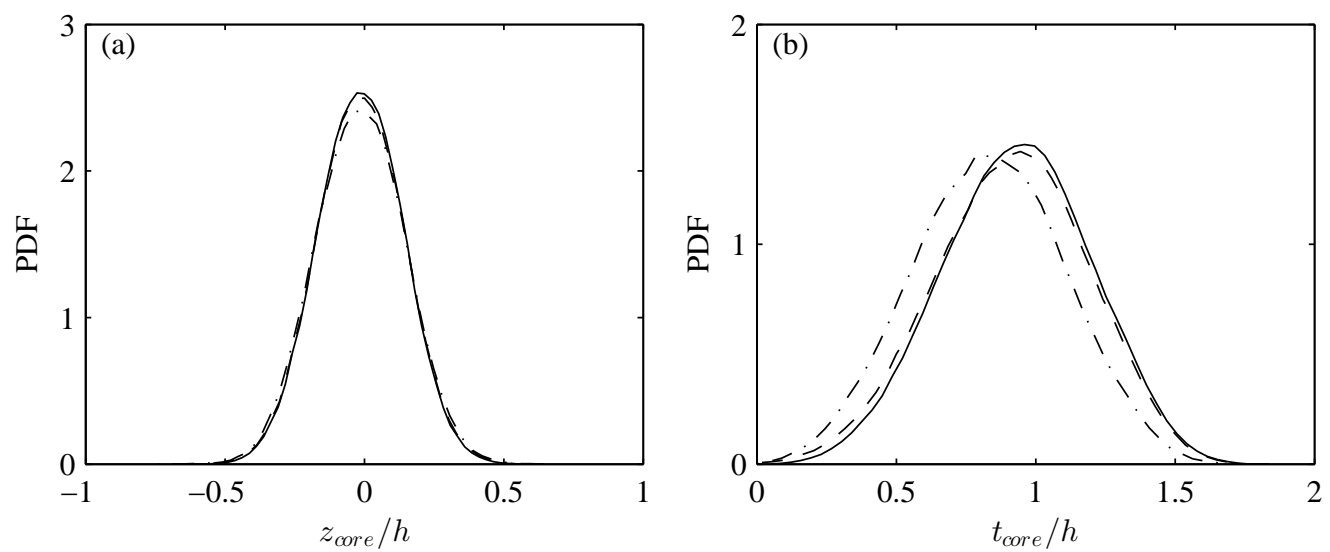

Figure 20. Distributions of (a) $z_{\text {core }}$ and (b) $t_{\text {core }}$ for 'inner' enveloped (dashed-dotted lines) and 'outer' enveloped (dashed lines for using the subset of dataset with continuous core and solid lines for using the whole dataset.) core at $R e_{\tau}=2000$.

factors including the flow quality and the noise level in the measurements. This means that, if the core was detected by means of other quantities and the same analysis in the paper were performed, the same conclusions would still be drawn.

\section{Appendix C. The effect of enveloping and selective usage of PIV data}

In situations where the core boundary folds back on itself, two different methods have been proposed for enveloping the core; 'inner', where the core boundary furthest from the wall is selected as the boundary, and 'outer', where the boundary closest to the wall is chosen. The 'inner' enveloped core boundary is preferred as a conservative method since it ensures that no outside region (non-core) is included, as previously mentioned. Here the distributions of $z_{\text {core }}$ and $t_{\text {core }}$ for the 'outer' enveloped core are compared with those for the 'inner' enveloped method. Figure 19(a) shows an example of outer enveloping, which can be compared with the inner enveloped core boundary in figure 6(b).

Figure 20 shows the PDF of $z_{\text {core }}$ and $t_{\text {core }}$ using the inner (dashed-dotted line) and outer (dashed line) enveloped core (for the subset of PIV datasets in which the core is 
continuous). It shows that the distribution of $z_{\text {core }}$ is not highly sensitive to the method of enveloping. However, $t_{\text {core }}$ is greater, on average, for the outer enveloped core as expected. A statistical analysis shows that the mean thickness of the outer enveloped core is about $13 \%$ greater than that for the inner enveloped core. However, the standard deviations of $z_{\text {core }}$ and $t_{\text {core }}$ do not depend significantly on the enveloping method.

Also in figure 20, we compare results for the 'outer' enveloped core in which either (i) the entire PIV dataset is used to calculate $t_{\text {core }}$ and $z_{\text {core }}$ (solid line) or (ii) only the subset of data in which the core is continuous for the entire PIV field of view is used (dashed line). When the core is discontinuous, and yet disconnected sections of the core overlap (figure 19b), we treat the overlapped part of the core as a single connected core (since they are likely to be parts of a single continuous core in the three-dimensional space as seen in figure 5(c). Thus, in these situations the cores are outer enveloped as shown in figure $19(\mathrm{~b})$. In figure 20 , the distribution of $z_{\text {core }}$ and $t_{\text {core }}$ from the subset of PIV dataset in which the core is continuous (dashed line) and the whole PIV dataset (solid line) agree reasonably well (the differences in both mean and standard deviation of $t_{\text {core }}$ and $z_{\text {core }}$ at all $R e_{\tau}$ are less than $4 \%$ ), verifying that disregarding the subset of PIV fields with discontinuous cores does not substantially impact the results.

\section{REFERENCES}

Adrian, R. J., Meinhart, C. D. \& Tomkins, C. D. 2000 Vortex organization in the outer region of the turbulent boundary layer. J. Fluid Mech. 422, 1-54.

del Alamo, J.C., Jiménez, J., Zandonade, P. \& Moser, R.D. 2004 Scaling of the energy spectra of turbulent channels. J. Fluid Mech. 500, 135-144.

Antonia, R. A., Teitel, M., Kim, J. \& Browne, L. W. B. 1992 Low-reynolds-number effects in a fully developed turbulent channel flow. J. Fluid Mech. 236, 579-605.

BAlAKumar, B J \& AdRian, R J 2007 Large- and very-large-scale motions in channel and boundary-layer flows. Phil. Trans. R. Soc. A 365, 665-681.

Bernal, P. \& Roshko, A. 1986 Streamwise vortex structure in plane mixing layers. J. Fluid. Mech 170, 499-525.

Bisset, D. K., Hunt, J. C. R. \& Rogers, M. M. 2002 The turbulent/non-turbulent interface bounding a far wake. J. Fluid Mech. 451, 383-410.

Brown, G. L. \& Roshko, A. 1974 On density effects and large structure in turbulent mixing layers. J. Fluid Mech. 64, 775-816.

Chauhan, K., Philip, J., De Silva, C., Hutchins, N. \& Marusic, I. 2014 The turbulent/nonturbulent interface and entrainment in a boundary layer. J. Fluid Mech. 742, 119-151.

Corrsin, S \& Kistler, A. L. 1955 Free-stream boundaries of turbulent flows. NACA Rep. 1244, 1033-1064.

Davidson, P. A. 2004 Turbulence: An Introduction for Scientists and Engineers. Oxford University Press.

Dean, R. B. \& Bradshaw, P. 1976 Measurements of interacting turbulent shear layers in a duct. J. Fluid Mech. 78, 641-676.

Drazin, P. G \& ReID, W. H. 1981 Hydrodynamic stability. Cambridge University Press.

Hunt, J. C. R., Eames, I., DA Silva, C. B. \& Westerweel, J. 2011 Interfaces and inhomogeneous turbulence. Phil. Trans. R. Soc. A 369, 811-832.

Hunt, J. C. R. \& Morrison, J. F. 2001 Eddy structure in turbulent boundary layers. Eur J. Mech. B Fluids 19, 673-694.

Jiménez, J., Hoyas, S., Simens, M. P. \& Mizuno, Y. 2010 Turbulent boundary layers and channels at moderate reynolds numbers. J. Fluid Mech. 657, 335-360.

KIM, J. 1989 On the structure of pressure fluctuations in simulated turbulent channel flow. $J$. Fluid Mech. 205, 421-451.

Kovasznay, L. S. G., Kibens, V. \& Blackwelder, R. F. 1970 Large-scale motion in the intermittent region of a turbulent boundary layer. J. Fluid Mech. 41, 283-325. 
LOFQUist, K. 1960 Flow and Stress Near an Interface between Stratified Liquids. Phys. Fluids 3, $158-175$.

Meinhart, C. D. \& Adrian, R. J. 1995 On the existence of uniform momentum zones in a turbulent boundary layer. Phys. Fluids 7, 694-696.

Monty, J.P. 2005 Development in smooth wall turbulent duct flows. PhD thesis, The University of Melbourne.

Monty, J. P., Hutchins, N., NG, H. C. H., Marusic, I. \& Chong, M. S. 2009 A comparison of turbulent pipe, channel and boundary layer flows. J. Fluid Mech. 632, 431-442.

NG, H.C. 2011 Experiments in smooth wall turbulent channel and pipe flows. PhD thesis, The University of Melbourne.

Roth, G. I. \& Katz, J. 2001 Five techniques for increasing the speed and accuracy of piv interrogation. Meas. Sci. Technol 12, 238-245.

Sabot, J. \& Comte-Bellot, G. 1976 Intermittency of coherent structures in the core region of fully developed turbulent pipe flow. J. Fluid Mech. 74, 767-796.

Semin, N. V., Golub, V. V., Elsinga, G. E. \& Westerweel, J. 2011 Laminar superlayer in a turbulent boundary layer. Tech. Phys. Letter 37, 1154-1157.

da Silva, C. B., Hunt, J. C. R., Eames, I. \& Westerweel, J. 2014 Interfacial layers between regions of different turbulence intensity. Ann. Rev. Fluid Mech. 46, 567-590.

DA Silva, C. B. \& Pereira, J. C. F. 2008 Invariants of the velocity-gradient, rate-of-strain, and rate-of-rotation tensors across the turbulent/nonturbulent interface in jets. Phys. Fluids 20, 055101.

de Silva, C. M., Baidya, R., Khashehchi, M. \& Marusic, I. 2012 Assessment of tomographic PIV in wall-bounded turbulence using direct numerical simulation data. Exp. Fluids $\mathbf{5 2}$ (2), 425-440.

Silva, R. A. \& DE Lemos, M. J. S. 2003 Turbulent flow in a channel occupied by a porous layer considering the stress jump at the interface. Int. J. Heat Mass Transfer 46, 5113-5121.

Teitel, M. \& Antonia, R. A. 1990 The interaction region of a turbulent duct flow. Phys. Fluids A 2 (5), 808-813.

Townsend, A. A. 1948 Local isotropy in the turbulent wake of a cylinder. Aust. J. Scientific Res. Series A: Physical Sciences 1, 161-174.

Townsend, A. A. 1956 The structure of turbulent shear flow, 1st edn. Cambridge University Press.

Westerweel, J., Hofmann, T., Fukushima, C. \& Hunt, J. C. R. 2002 The turbulent/nonturbulent interface at the outer boundary of a self-similar turbulent jet. Exp. Fluids 33, $873-878$ 